\title{
Colloidal nanocrystals for photoelectrochemical and photocatalytic water splitting
}

\author{
Chethana Gadiyar, ${ }^{\mathrm{a}}$ Anna Loiudice, ${ }^{\mathrm{a}}$ Raffaella Buonsanti ${ }^{\mathrm{a} *}$ \\ ${ }^{a}$ Department of Chemical Sciences and Engineering, École Polytechnique Fédérale de Lausanne, Sion \\ 1950, Switzerland. \\ *Corresponding author email: raffaella.buonsanti@epfl.ch
}

\begin{abstract}
Colloidal nanocrystals (NCs) are among the most modular and versatile nanomaterial platform to study emerging phenomena in different field thanks to their superb compositional and morphological tunability. A promising, yet challenging, application involves the use of colloidal NCs as light absorber and electrocatalyst for water splitting. In this review we discuss how the tunability of these materials is ideal to understand the complex phenomena behind storing energy in chemical bonds and to optimize performance through structural and compositional modification. First, we describe colloidal synthesis approaches that offer a high degree of control over single material NCs and NC heterostructures, including examples on the role of the ligands to modulate size and shape. Next, we focus on the use of NCs as light absorbers and catalysts to drive both the hydrogen evolution reaction (HER) and the oxygen evolution reaction (OER) along with some of the challenges related to the use of colloidal NCs as model systems and/or technological solutions in water splitting. We conclude with a broader prospective on the use of colloidal chemistry for new material discovery.
\end{abstract}

\section{Introduction}

Much progress has been made since Honda and Fujishima discovered that $\mathrm{TiO}_{2}$ is capable of splitting water in 1972 [1]. However, the storage of sunlight into chemical bonds, namely artificial photosynthesis, cannot be efficiently completed yet with robust and Earth-abundant materials. The combination of modeling and experiments have shown that higher efficiencies are achieved with dual tandem absorbers design, instead of one single semiconductor material [2,3]. Dual tandem absorber configurations include two properly matched light absorbers, that generate the photovoltage needed to split water, and catalysts specifically optimized for water oxidation or reduction, that are interfaced with the semiconductor light absorbers $[2,3]$. Yet, the coupling of photons and electrons, protons and electrons and even the catalytic mechanism behind water splitting have not been fully elucidated. The knowledge gap becomes even larger when looking at the carbon dioxide reduction reaction.

Improving the performance of sustainable solar-to-fuels conversion requires probing and understanding these nanoscale phenomena with advanced scientific techniques. This understanding must then be translated into control of the functionality and performance of the materials and chemistry that govern the water splitting and the $\mathrm{CO}_{2}$ reduction reactions. Here, the ability to produce materials with tailored structure and composition is key to derive meaningful structure/function relations. Conventional synthetic techniques (i.e. solid state reactions, sol-gel processing, electrochemical deposition) often provide 
samples that are heterogeneous in nature. Because the materials can undergo unknown structural changes during the photoelectrochemical and electrochemical testing, significant effort are expected to understand what makes the light absorber, the catalyst and the combination of both efficient. Precise engineering and manipulation of the active materials is instead needed to elucidate the relevant structural parameters governing their catalytic or photocatalytic behaviour.

Colloidal nanocrystals (NCs) are among the most modular and versatile nanomaterial platform to study emerging phenomena in different field due to their unprecedented compositional and morphological tunability [4-25]. A collection of transmission electron microscopy (TEM) images of various NCs is shown in figure 1 . They are constituted by an inorganic core, with tunable composition, size and shape, and a shell of amphiphilic surfactants or ligands. The latter are key in controlling the NC morphology. Furthermore, they guarantee the dispersability of colloidal NCs in different solvent, thus allowing their deposition onto various substrates or their integration into various matrices (i.e. inorganic or polymeric). These processes are not possible with powder-form nanoparticulate or NCs synthesized by chemical vapor deposition (CVD) and molecular beam epitaxy (MBE). 

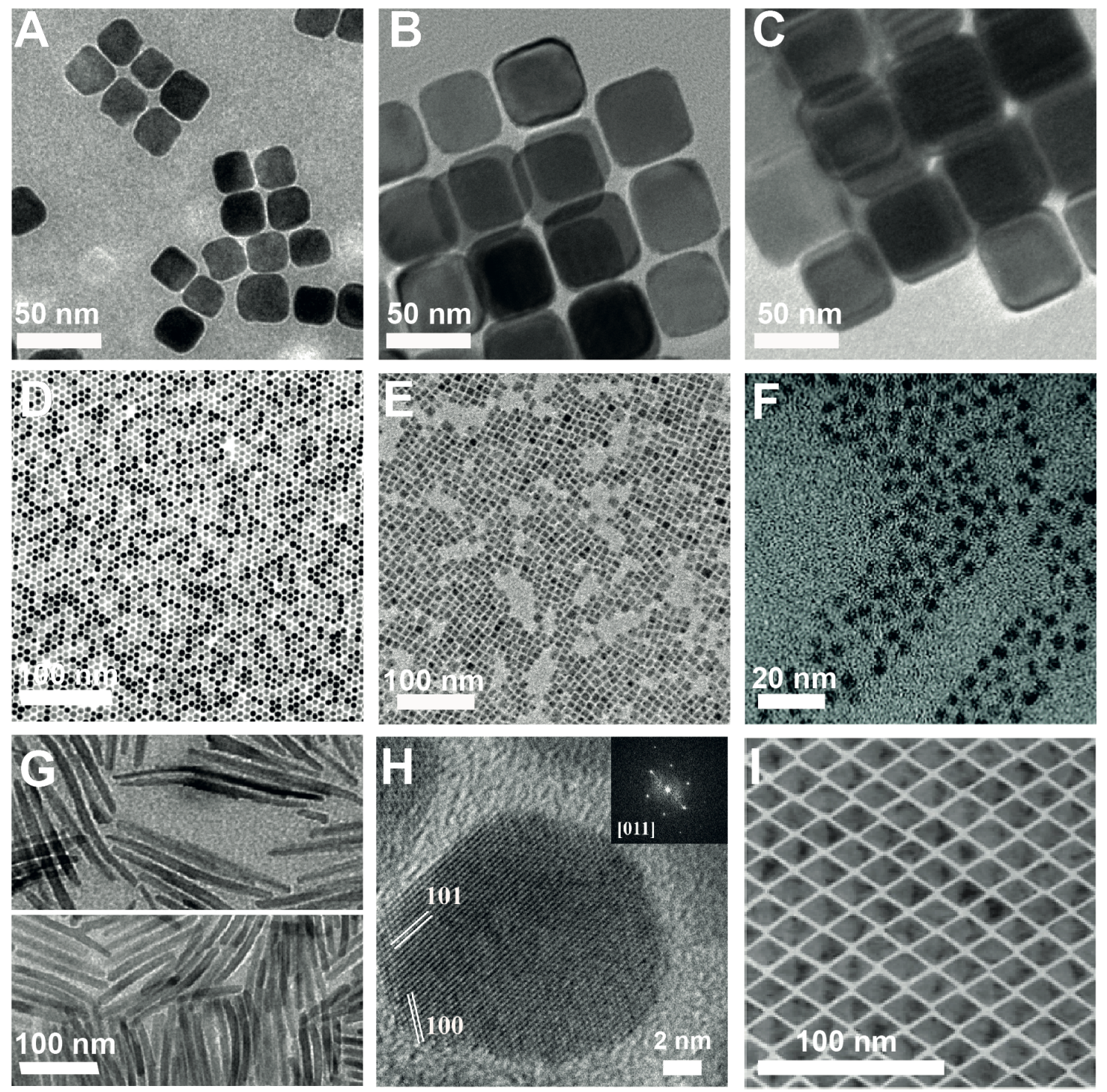

Figure 1: Overview of TEM images of colloidal NCs: (A-B) Schematic and TEM images of $\mathrm{Cu}$ nanospheres and nanocubes [20]; (C) $\mathrm{Cu}_{2} \mathrm{ZnSnS}_{4} \mathrm{NCs}$ [21]; (D) $\mathrm{CsPbBr}_{3} \mathrm{NCs}$ (E) Ag-In-Se NCs [23]; (F) brookite $\mathrm{TiO}_{2}$ nanorods [9]; (G) Al-doped ZnO NCs [24]; (H) $\mathrm{DyF}_{3}$ rhombohedral nanoplates [25]. Reprinted from [20] with permission from John Wiley and Sons, copyright 2016. Reprinted with permission from [9,21,24], copyright 2008, 2011, 2016 American Chemical Society. Reproduced from [23] with permission of The Royal Society of Chemistry. Reprinted by permission from Macmillan Publishers Ltd: Nature Chemistry [25], copyright 2013.

The use of colloidal NCs in various catalytic reactions, such as CO oxidation and oxygen reduction, has demonstrated their huge potential to function as model systems to propose new catalytic schemes or to elucidate the role of defects, catalyst/support interface, crystalline phase [26-30]. As for the water splitting reactions, to date most of the work with colloidal NCs has focused on heterostructured NCs comprising a metal catalyst coupled with a semiconductor light absorber to drive the sacrificial hydrogen production. 
While long term stability still remains to be solved, compelling efficiencies have been achieved. More recently, colloidal NCs, mostly belonging to the phosphide family, have been used as hydrogen evolution catalyst. Far less studies have focused on the oxygen evolution reaction, yet their number is increasing. Motivated by the growing interest in the field by the nanocrystal community, we have decided to give an extensive overview of the state-of-art work relative to the application of colloidal NCs for photocatalytic (no bias applied) and photoelectrochemical (bias applied) water splitting. The aim is to help to identify the challenges and the research areas which deserve more attention so to direct future efforts towards those. We omit to review studies on nanocrystalline materials prepared by other routes and used in suspensions, instead of uniform colloidal solutions. Therefore, the work from the Domen and the Osterloh groups are not discussed in the present contribution [31-35]. First, we briefly overview single material NCs, NC heterostructures and some of the approaches to synthesize them. Next, we focus on the use of NCs as light absorbers and catalysts to drive both the hydrogen evolution reaction (HER) and the oxygen evolution reaction (OER). We also describe some of the challenges related to the use of colloidal NCs as model systems and/or technological solutions in water splitting Finally, future opportunities of using colloidal chemistry for new material discovery are succinctly highlighted.

\section{General concepts in colloidal nanocrystal synthesis \\ 2.1 Single-material nanocrystals}

Colloidal synthesis is a surfactant-assisted approach which has proven to afford a superior tuning of the size, the shape and the composition of nanocrystalline materials compared to other techniques [4-25,36,37]. In colloidal synthesis, the growth medium is a liquid mixture of ligands and surfactants and the whole synthesis process is usually carried out under inert atmosphere, as sketched in figure 2A. Some of the surfactants commonly used include alkylthiols, amines, carboxylic and phosphonic acids, phosphines, phosphine oxides and various coordinating solvents, such as ethers or alkenes [4-25,36,37]. The general scheme of a colloidal synthesis involves thermally activated reaction of organometallic precursors (that carry the atomic species necessary to build the desired material) in the presence of selected surfactants (figure 2B). Once the synthesis is activated, the highly reactive species that are generated, commonly referred to as the "monomers", induce the nucleation of NCs and sustain their subsequent enlargement. The surfactants play several key roles during the synthesis. Indeed, they form complexes with the monomers, dictating their actual chemical potential in solution [9-13]. Simultaneously, they participate in an adsorption/desorption dynamics on the surface of the growing clusters, which prevents aggregation and uncontrolled growth. Mechanistic studies have shown that monodisperse NCs are produced when a burst of nucleation that enables separation of the nucleation and growth processes is combined with diffusion-controlled growth. Satisfactory tailoring of the NC size and size distribution can be achieved by balancing the relative depletion of monomers between the nucleation and the growth stages either with the aid of suitable techniques (i.e. the "hot injection"), or profiting from the particular reactivity of the system (i.e. "delayed nucleation"), or exploiting digestive-ripening [5,7,38]. It is also worth mentioning that surfactants can affect the specific surface energy of the growing NCs, which has important implications in the tuning of their shape $[5,7,8]$. Indeed, facet-preferential li 


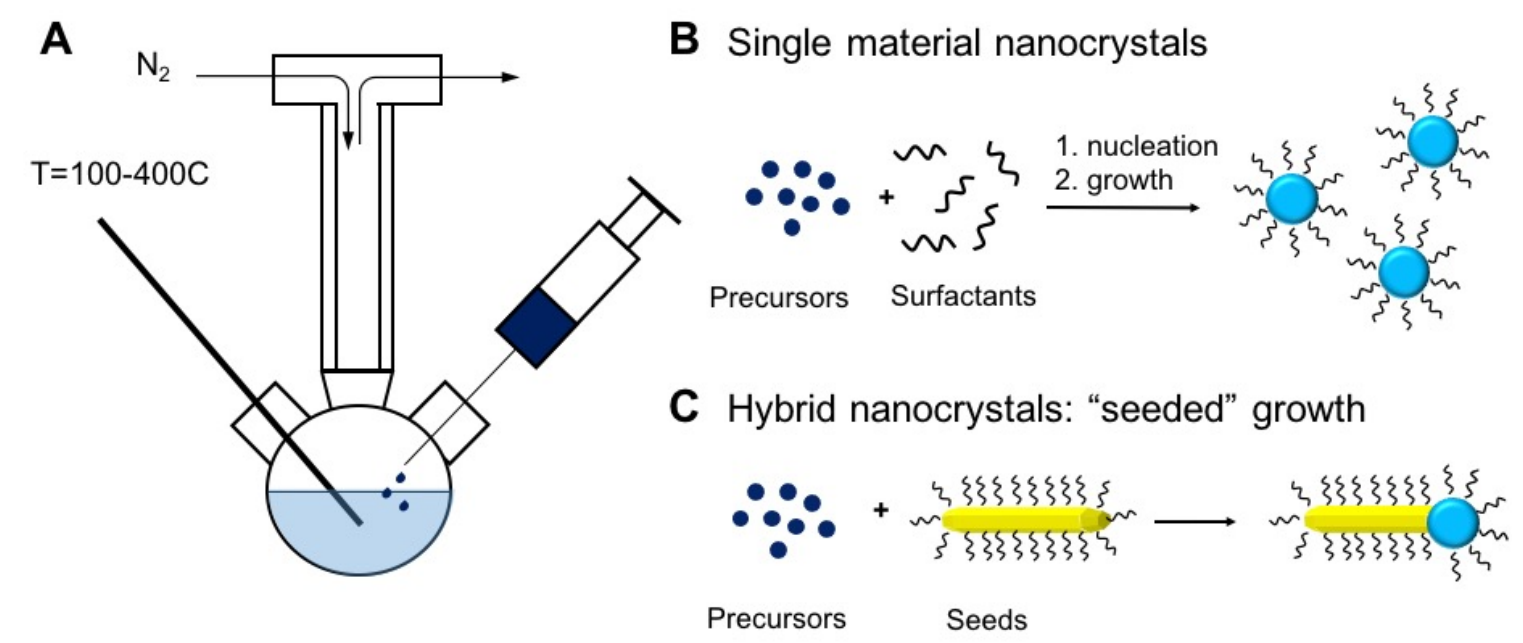

Figure 2: Schematic of (A) the setup used for colloidal synthesis consisting of a threenecked flask, a heating mantle and a condenser; (B) the synthesis of single material NC; (C) the synthesis of hybrid NCs by seeded growth approach.

gand adhesion can modify the relative growth rates along the various crystallographic directions and/or can favour selective elimination of unstable surfaces by triggering oriented attachment of particles along defined crystallographic directions. In the absence of additional circumstances that can interrupt growth symmetry (e.g., formation of soft micelle templates, the presence of foreign particle catalysts, or the application of external electric or magnetic fields), surfactants remain mostly responsible for the formation of NCs in a variety of anisotropic shapes, such as cubes, polyhedrons, rods, wires, polypods, and rings, as shown in figure 1.

\subsection{Nanocrystalline heterostructures or hybrid nanocrystals}

$\mathrm{NC}$ heterostructures or hybrid NCs (HNCs) are multi-material nano-objects, consisting of two or more different material domains, often interconnected through epitaxial interfaces [14-19,39-43]. One of the most common synthetic approaches to these multicomponent nanomaterials is "seeded growth". "Seeded growth" relies on performing preferential heterogeneous nucleation and growth of a second material on preformed $\mathrm{NC}$ seeds, as shown in figure 2C. Other synthetic strategies include cation exchange, phase segregation, coalescence of an initially amorphous shell. Figure 3 gives examples of HNCs with different topologies and highlights once again the high level of control achievable by colloidal chemistry. These different topologies are dictated primarily by the lattice mismatch between the two domains; however, different synthetic strategies can impact topology as well. As one example, by tuning the reactivity and/or concentration of the monomer of the second material, it is possible to decide whether, starting from one rod-like semiconductor seed, either both ends of the rod, only one end or the longitudinal side is decorated with the second domain [15,39]. It is helpful to consider that the creation of HNCs by seeded growth in solution phase shares some similarities with the more traditional heteroepitaxial deposition processes accomplished by MBE and CVD techniques, in terms of growth mode $[44,45,46]$. However, what clearly distinguishes colloidal seeded growth from MBE/CVD approaches is the presence of surfactants that indeed alter the surface energy term considerably. This is one of the reasons why strain- 
related hetero-epitaxial growth constraints can be greatly overcome by colloidal chemistry. As an example, epitaxial interfaces have been observed in $\mathrm{TiO}_{2}-\mathrm{Fe}_{2} \mathrm{O}_{3}$ even with a $13 \%$ lattice mismatch [40].

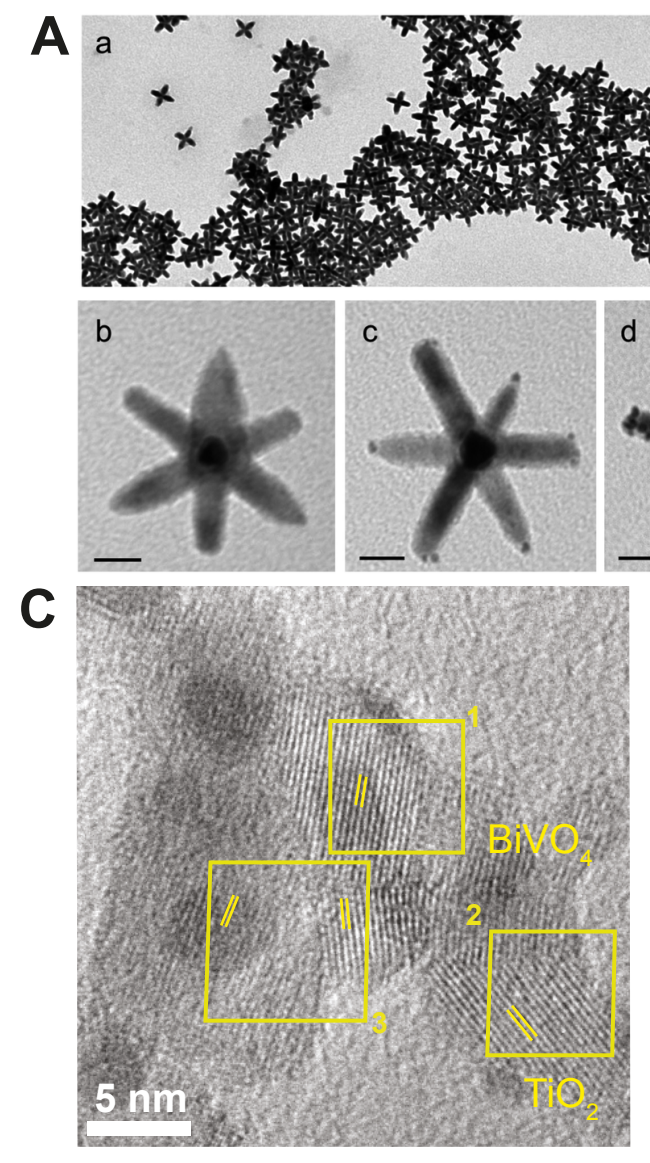

$\mathbf{E}$

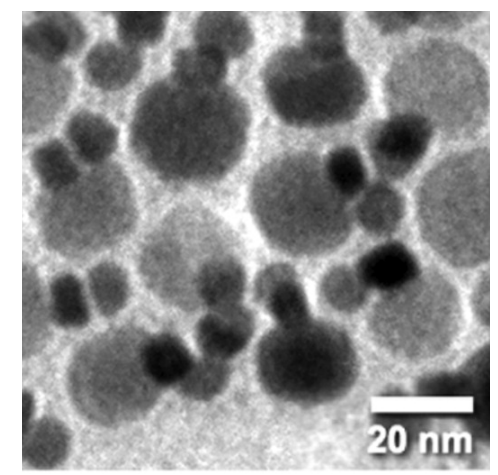

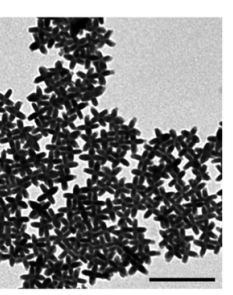
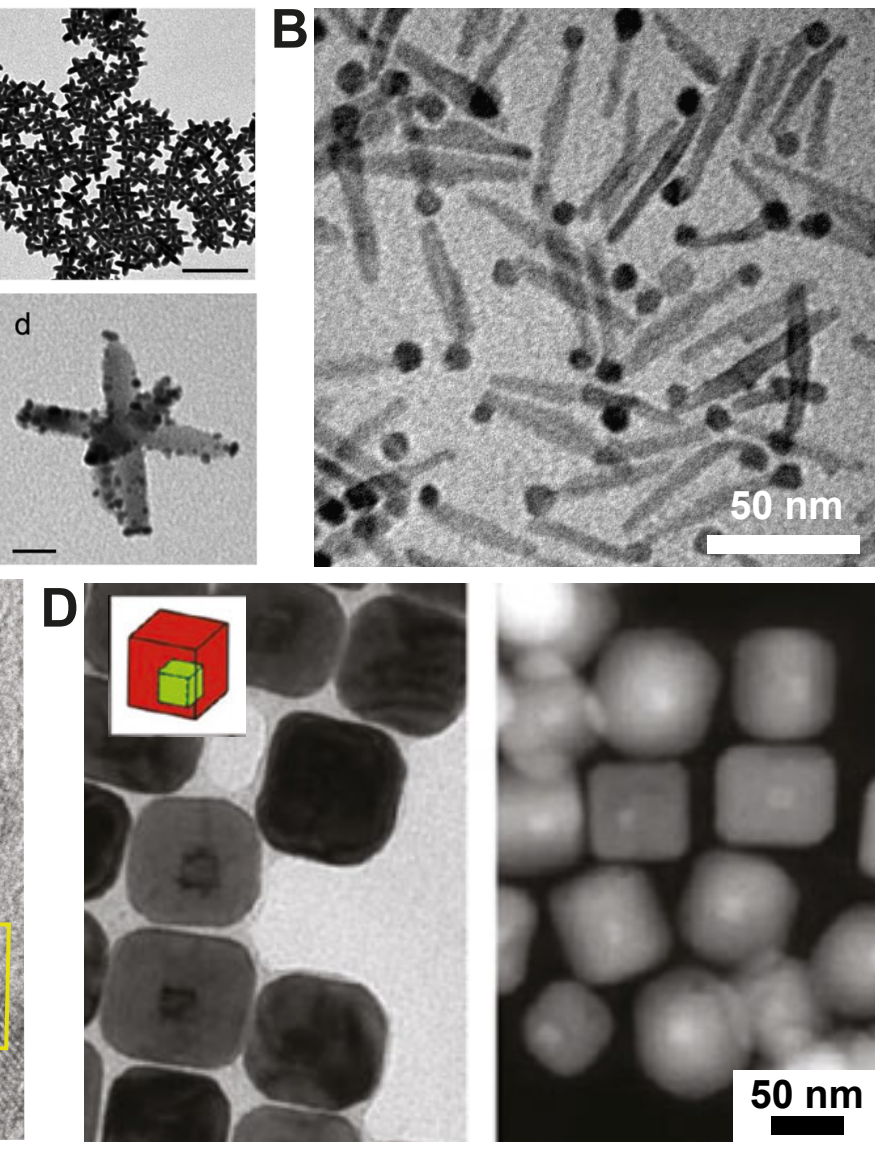

$\mathbf{F}$
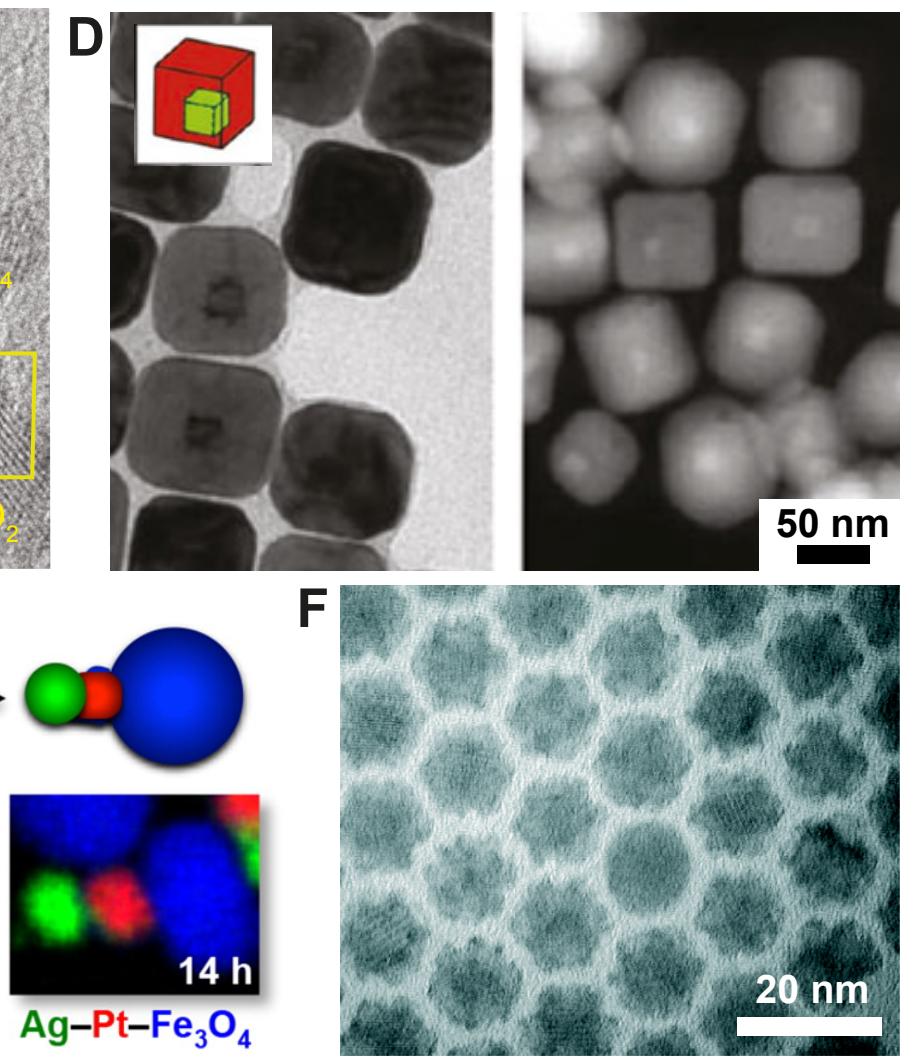

Figure 3. TEM examples of hybrid NCs with different topological distribution of their component material sections. (A) Pt-decorated CdSe@CdS octapod NCs:(a) TEM image of an ensemble of CdSe@CdS octapod nanocrystals, the scale bar is $200 \mathrm{~nm}$. Representative images of (b) a single Pt-free octapod nanocrystal, (c) a single Pt-tipped octapod nanocrystal and (d) a Pt-covered octapod nanocrystal. Scale bars in (b), (c) and (d) are $20 \mathrm{~nm}$ [14]; (B) $\gamma-\mathrm{Fe}_{2} \mathrm{O}_{3} / \mathrm{TiO}_{2} \mathrm{NC}$ hybrids [15]; (C) $\mathrm{BiVO}_{4} / \mathrm{TiO}_{2}$ heterostructures [16]; (D) Pd@Cu core@shell nanocubes [47]; (E) Ag-Pt-Fe $\mathrm{O}_{4}$ trimers [17]; (F) RuRh$\mathrm{Cu}_{2} \mathrm{~S}$ nanocages [18]. Reproduced from $[14,18]$ with permission of The Royal Society of 
Chemistry. Reprinted with permission from [15-17,47], Copyright 2010,2015, 2015,2012 American Chemical Society.

\section{Colloidal nanocrystals as light absorbers}

In recent years, the reports on colloidal NCs used as light absorbers to drive the water splitting have notably increased, with the studies on HER greatly outnumbering those on OER. While in this review we will limit our discussion to fully inorganic colloidal HNCs, we notice that light absorber NCs have been successfully coupled with enzymes, specifically iron hydrogenases, and molecular species as co-catalyst for hydrogen generation [48-50]. Studies relative to dye reduction or oxidation are also not included in this contribution.

\subsection{Colloidal nanocrystals as light absorbers for HER}

The general approach to drive HER with colloidal NCs has been to decorate II-VI semiconductor NCs (or quantum dots, QDs) with metallic nanoparticles. The latter promote charge separation of the photogenerated carriers and also act as the site for hydrogen generation. Most of these semiconductor/metal HNCs have been tested as phototocatalysts dispersed in solution, with the exception of few examples [51,52]. To perform the testing in aqueous solutions, the native hydrophobic ligands functionalizing the NC surface are exchanged with shorter hydrophilic ligands, the most common ones being mercaptoundecanoic acid (MUA) with some examples using cysteine and mercaptopropionic acid (MPA). Several reviews on semiconductor-metal colloidal NC heterostructures for HER have been published in the past years [53-55]. We refer to them for more details, especially with regards to studies of charge carrier dynamics which are not included here. For sake of clarity, literature has been grouped by classes of the light absorbing semiconductor materials. It should be noted that most of the papers reviewed in this section reports performance as external or apparent quantum yield (EQE or AQE) of hydrogen evolution and uses a visible laser as the light source. The AQE is defined as two times the rate of collection of $\mathrm{H}_{2}$ to the incident photon flux, given by equation (1). We have reported the $\mathrm{AQE}$ values at the irradiation wavelength, when data were available, so to have a measureable comparison between different photocatalysts under similar experimental conditions. Other ways to report the efficiency for photocatalytic hydrogen generation include quantum yield (QE) and solar-to-hydrogen (STH) energyconversion efficiency $[31,56]$. QE is the ratio of the number of "reacted" electrons per number of absorbed photons, as shown in equation (2). Instead, the STH indicates the conversion of the energy in the incident solar irradiation to chemical bonds as $\mathrm{H}_{2}$ through oxidation of water to $\mathrm{O}_{2}$, given by equation (3) [31,56]. In equation (3), $237000 \mathrm{~J} / \mathrm{mol}$ is the standard Gibb's free energy of water splitting reaction.

$$
\begin{gathered}
A Q E=\frac{\text { Number of reacted electrons }}{\text { Number of incident photons }}=\frac{2 \times\left(\mathrm{mol} \mathrm{H}_{2} / \mathrm{s}\right)}{\text { Number of incident photons }} \\
Q E=\frac{\text { Number of reacted electrons }}{\text { Number of absorbed photons }}
\end{gathered}
$$




$$
S T H=\frac{\left(\mathrm{mol} \mathrm{H}_{2} / \mathrm{s}\right) \times(237000 \mathrm{~J} / \mathrm{mol})}{P_{\text {inc }}\left(\mathrm{W} / \mathrm{cm}^{2}\right) \times \operatorname{Area}\left(\mathrm{cm}^{2}\right)}
$$

CdS and CdSe NCs.

$\mathrm{CdS} / \mathrm{Pt}$ and $\mathrm{CdSe} / \mathrm{Pt} \mathrm{HNCs}$ are the first and among the most studied systems so far (figures 4A,B,D). CdS and CdSe nanorods (NRs) have been largely preferred to spherical NCs as they have a lower exciton binding energy and offer the possibility to control the distance between reaction sites. Tailored variation of the NR length, diameter and shape as well as Pt domain size have enabled correlations between the structural parameters and the efficiencies [57-59]. As one example, Berr et al. have showed that the hydrogen evolution rate does not benefit from larger Pt domains $(4.8 \mathrm{~nm})$ on CdS NRs when compared to subnanometer sized Pt clusters [58]. Similar results were found for Ptdecorated CdSe tetrapods reported by Sung et al. [59]. The higher surface-to-volume ratio of the small clusters and probably different charge separation kinetics have been identify to play an important role in the higher photocatalytic efficiencies compared to the particles. These findings suggest that efficient hydrogen production utilizing II-VI nanostructures with reduced amounts of Pt is possible. In addition to Pt as metallic cocatalyst, a few studies on $\mathrm{CdSe} / \mathrm{Au}$ have been reported [60,61]. For example, using a well-defined model system of $\mathrm{CdS} / \mathrm{Au} \mathrm{NRs}$, Ben-Shahar et al. have studied the effect of the gold size tip on the photocatalytic function, including the charge transfer dynamics and the $\mathrm{H}_{2}$ production efficiency [62]. They found a non-monotonic behavior with size of the gold tip, leading to an optimal metal domain size. The optimal value is explained in terms of competing processes where for small tips the hydrogen evolution QY is mainly determined by the rate of electron injection to the metal tip, whereas for large tips it is determined mainly by the water reduction kinetics on the metal surface. These two limits show opposite dependence on the metal domain size, leading to an optimal value as explained by a minimalist and general kinetic model [62].

A few examples including a non-noble metal catalyst have been reported [63-65]. Cao et al. have revealed the potential of $\mathrm{CdS} / \mathrm{Ni}_{2} \mathrm{P}$ and $\mathrm{CdS} / \mathrm{Co}_{2} \mathrm{P}$ by measuring their activity in an aqueous lactic acid solution [63]. In particular they have achieved a AQE of hydrogen generation of $6.8 \%$ under LED light $(\lambda>420 \mathrm{~nm})$ by physically mixing CdS NRs and $\mathrm{Co}_{2} \mathrm{P}$ nanoparticles [63]. As for the $\mathrm{Ni}_{2} \mathrm{P}$ they report only rate of hydrogen generation [64]. Zhukovskyi et al have decorated $\mathrm{CdS}$ nanosheets with $\mathrm{Ni}$ and have been able to achieve AQE up to $25 \%$ under laser irradiation at $405 \mathrm{~nm}$ for the optimal catalyst loading [65].

Iron-based alternatives are especially attractive because $\mathrm{Fe}$ is the most abundant transition metal. Cheng et al. have successfully demonstrated a low-cost yet highly efficient and stable semiconductor $\mathrm{CdS} / \mathrm{FePt}$ photocatalytic system [66]. An AQE of over $35 \%$ at $520 \mathrm{~nm}$ was measured. This value was 3 -fold higher than that of the control $\mathrm{CdS} / \mathrm{Pt}$ system.

Because photo-oxidation is an issue for stability of semiconductor NCs, the photo-holes need to be quickly extracted from the surface. Thus, a hole scavenger (or electron donor) is used in all the reports. While alcohols are the most common, it is clear that the redox level of the scavenger with respect to the valence band of the semiconductor NCs will 
impact the charge transfer kinetics and therefore the quantum efficiency for hydrogen generation. A nice study by Berr et al. illustrates the positive effect of the increased redox potential of the hole scavenger $\left(\mathrm{MeOH}<\mathrm{EDTA}^{4-}<\mathrm{TEA}<\mathrm{SO}^{3-}\right)$, which facilitates a faster hole scavenging, on both the activity and the stability of the CdS/Pt hybrids (figures 4A,D,E) [67]. In particular the authors compare the hydrogen evolution in function of the time during exposure under illumination. They found a correlation between the oxidation potential of the hole scavenger and the stability of the photocatalyst. With TEA and EDTA ${ }^{4-}$ the amount of hydrogen product dropped from a maximum of $2.5 \mu \mathrm{mol}$ and $0.4 \mu \mathrm{mol}$ to $0.6 \mu \mathrm{mol}$ and $0.07 \mu \mathrm{mol}$ after $4 \mathrm{~h}$ of illumination, respectively. The hydrogen generated in the presence of $\mathrm{MeOH}$ was below the detection limit. The best stability was measured in the presence of $\mathrm{SO}^{3-}$ as hole scavenger. The conclusion of this study was that the higher the oxidation potential is, the more efficient the hydrogen production and the more stable the nanorods are.

High $\mathrm{pH}$ has been demonstrated to increase efficiency and stability of CdS/Pt and of CdS/Ni hybrid NCs $[68,69]$. The authors have demonstrated that although the yield decreases to approximately $20 \%$ after $80 \mathrm{~h}$, it then remains stable for up to $220 \mathrm{~h}$. This means that at high $\mathrm{pH}$ the system is protected against photo-oxidation. This happens because of the redox couple ${ }^{-} \mathrm{OH} / \mathrm{OH}$ operating as a shuttle between the surface of the $\mathrm{NCs}$ and the ethanol scavenger. As illustrated in figure 4F, the authors suggest that instead of a direct oxidation of ethanol by the photoexcited hole, the small sized hydroxyl anion, present in large quantities in highly alkaline conditions, easily diffuse to the NR surface where it gets oxidized. The AQE of $\mathrm{H}_{2}$ generation overcomes $50 \%$ under $446 \mathrm{~nm}$ laser illumination of a dispersion of Ni-decorated, cysteine stabilized CdS NRs in the presence of ethanol as a sacrificial agent. This work establishes the record efficiencies for CdS NCs used as photocatalysts to drive hydrogen evolution.

\section{CdSe@CdSHNCs.}

In addition to scavenging them with a sacrificial agent, the holes can be confined to a part of the nanostructure that is acting like a trap. The use of heterostructured $\mathrm{CdSe} / \mathrm{CdS}$ nanointerfaces has been demonstrated to further increase the QY of hydrogen production through the spatial separation of charges in nonadjacent domains. In CdSe@CdS/metal HNCs, the photogenerated charge carriers are separated with hole in the core (CdSe) and electrons in the shell $(\mathrm{CdS})$, that are eventually transferred to the metal domain to generate hydrogen.

Amirav et al. have assembled heterostructures including $\mathrm{CdSe} @ \mathrm{CdS}$ asymetric core@shell NRs functionalized with Pt nanoparticles (figure 4B)[70]. What is interesting about the asymmetric dot@rod core@shell is that the extent of charge separation can be modulated by the length NR shell, and so it is the efficiency of hydrogen production. As a matter of fact, the hydrogen production of the CdSe@CdS/Pt was almost 30 times higher than $\mathrm{CdS} / \mathrm{Pt}$ for the same length of CdS with a maximum AQE of $20 \%$ at $450 \mathrm{~nm}$ reached for $60 \mathrm{~nm} \mathrm{CdS}$ rod with a CdSe core of $2.3 \mathrm{~nm}$. Wu et al. have evaluated the performance of MUA-capped CdS/Pt and CdSe@CdS/Pt NRs in aqueous solutions using methanol or sulfite as hole scavenger [71]. The relative behaviour of these two NR-based systems varied with the nature of the hole scavenger: higher QE (2\% versus $0.8 \%)$ was observed for $\mathrm{CdSe} @ \mathrm{CdS} / \mathrm{Pt}$ when methanol was used; instead, CdS/Pt was more efficient than $\mathrm{CdSe} @ \mathrm{CdS} / \mathrm{Pt}(10 \%$ vs $3 \%)$ in the presence of sulfite. Utilizing ultrafast transient 
absorption spectroscopy the authors have measured the transfer rate of the electrons to the Pt domain and of the holes to the surface. They found that the electron transfer efficiency to the Pt tip is near unity for both systems. Instead, the hole transfer rate was slower in CdSe@CdS due to the hole confinement within the CdSe core that is harder to access by the electron donor. The hole transfer rates likely depend also on the interaction of the electron donor with the surface ligands and the accessibility of surface sites. The authors found a positive correlation of the observed hole transfer rates to the scavenger with the steady-state $\mathrm{H}_{2}$ generation $\mathrm{QE}$, indicating that hole transfer was a key efficiencylimiting step [71]. Despite the importance of hole transfer in determining both efficiency and stability, electron transfer studies outnumber hole studies. Different groups are investigating this aspect into more details for the CdSe@CdS system [72,73]. Examples are given below in section 5.1.

As an alternative to Pt as HER catalyst, recent works have studied the effect of different metallic and bimetallic co-catalysts on the CdSe@CdS rods tip to improve the efficiency for the photocatalytic water splitting reduction half reaction [60,74,75]. Nakibli et al. have compared the effect of Au, Pt, Au@Pt and Au-Pt deposited simultaneously [74]. Rods decorated with Au cocatalyst showed very low activity, compared with the Pt cocatalyst. When the Au tip was covered with Pt in a core@shell configuration, the performance were improved compared to pure $\mathrm{Pt}$, with the best performance obtained when the Au-Pt were deposited simultaneously. The suggested reason relies on the fact that the injection of the photoinduced electrons from CdSe into the Au tip is faster than their injection into the Pt tip. At the same time, only Pt can completely extract the excited electrons from the semiconductor NRs [76]. Thus, building a golden gate through which electrons can migrate from the semiconductor photocatalyst to the Pt cocatalyst islands results in increased activity for the water reduction half reaction. The same authors demonstrated that the synergistic effects of $\mathrm{Au}$ and $\mathrm{Pd}$ are beneficial for both the overall stability and the efficiency of the photocatalysts [77]. High-resolution TEM techniques were used to unravel the effects of structural changes at the atomic scale on the overall function and durability of heterostructured photocatalysts. The authors showed that the mobility of $\mathrm{Pd}$ atoms has a detrimental effect on the stability of the nanostructures due to atomic scale corrugation processes and that the Au serves as a physical barrier against $\mathrm{Pd}$ migration [77]. More recently, the Amirav group has published a study where the Pt catalyst is substituted with Ni domains. Similar AQE (23\%) were achieved respect to that for Pt (27\%) [74]. Ehrat et al. have also decorated core@shell CdSe@CdS with Ni [78]. They have shown that the hydrogen generation rate increases with the shell thickness, but decreases with the increasing core size. Interestingly, they have found that the photoluminescence quantum yield and lifetime follow the same trend. The correlation between the photocatalytic performance and photoluminescence lifetime is to be expected because they both rely on efficient charge separation [78].

Very recently, the record AQE of 100\% for CdSe@CdS core@shell NRs has been achieved by Kalisman et al. when the system was tested at very basic $\mathrm{pH}$ illuminated with a LED at $455 \mathrm{~nm}$ [79]. This result was attributed to the presence the hydroxyl anionradical redox couple operating as a shuttle to relay the holes, in a similar manner to the work by Simon et al cited above [69]. The authors also demonstrated 44h stability under non-continuous illumination. This work highlights once more the importance of efficiently extracting the holes to boost efficiencies. 

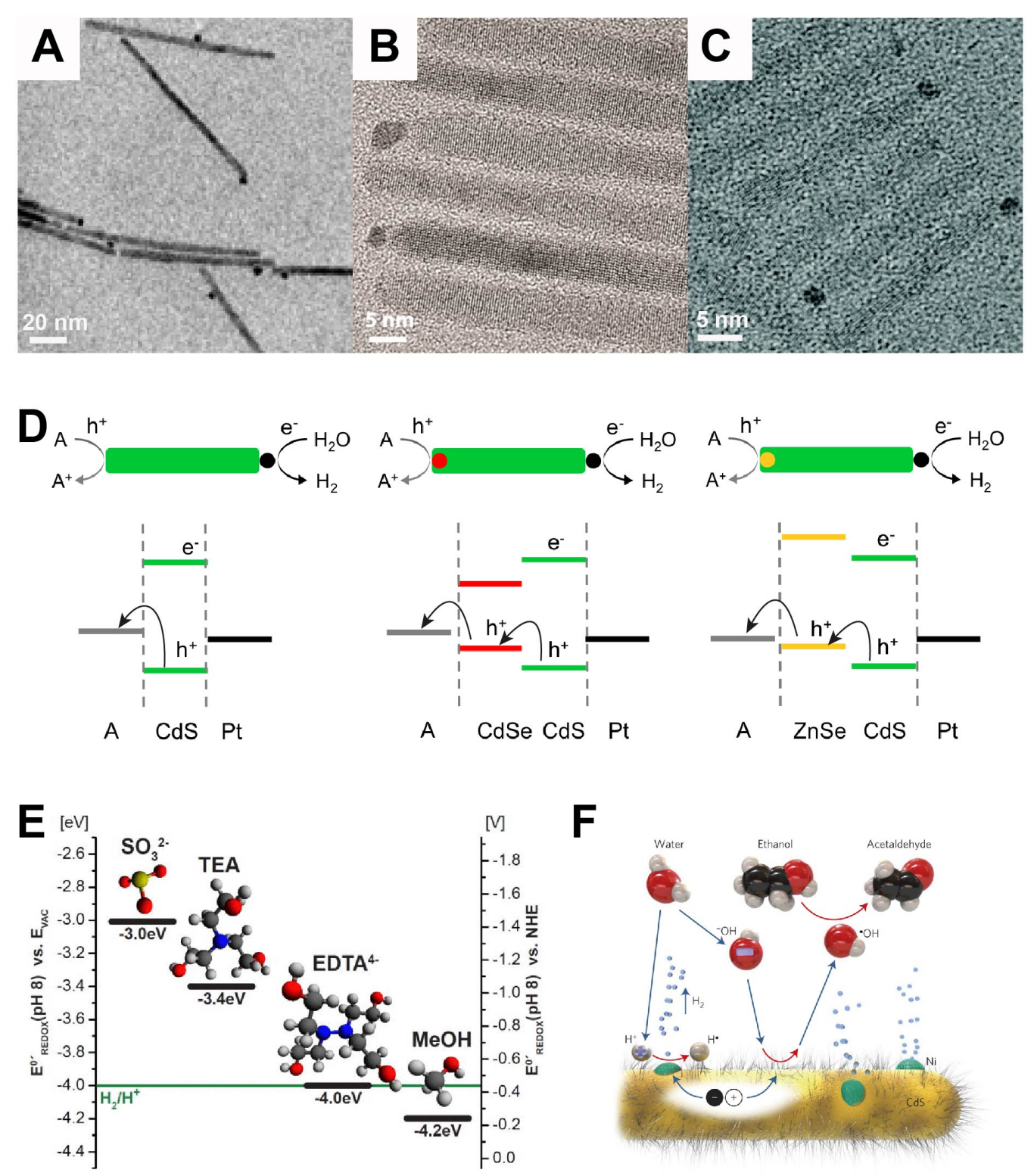

Figure 4. Semiconductor/metal hybrid NCs for HER. TEM images of (A) metal tipped CdSe nanorods [81], (B) Pt tipped CdSe@CdS nanorods [79], (C) Pt tipped ZnSe@CdS nanorods [80], respectively. (D) Schematic representation of the relative band position for the corresponding HNCs in figure A-C illustrating the hole and electron path after illumination toward the scavenger (A) or catalyst (Pt), respectively. (E) Schematic plot of the energy levels of the electrons of $\mathrm{SO}_{2}{ }^{-3}$, TEA, EDTA ${ }^{4-}$, and $\mathrm{MeOH}$ that are involved in the reduction of the photohole vs. vacuum and on normal hydrogen electrode (NHE) scale [81]. (F) Scheme of the photocatalytic water reduction by CdS-Ni nanorods (cysteine-stabilized) at alkaline conditions. The photo-excited holes oxidize hydroxyl anions that, as a radical, carry away the positive charges and in turn oxidize ethanol to acetaldehyde. The blue arrows denote movement of the species; the red arrows denote a redox reaction [69]. Reproduced from [79,80], Copyright 2016,2011 American Chemical Society. Reprinted from [81], with the permission of AIP Publishing. Reprinted by 
permission from Macmillan Publishers Ltd: Nature Materials [69], copyright 2014.

Other Cd-based semiconductor NCs.

To tune the localization of photoinduced holes Acharya et al have synthesized asymmetric core@shell HNCs including ZnSe@CdS/Pt and ZnTe@CdS/Pt (figures $4 \mathrm{C}, \mathrm{D})[80]$. In this study the authors evidenced the role of the NC ligands in mediating the hole extraction from the NC surface. The comparison of photocatalytic activity in ZnSe@CdS/Pt and ZnTe@CdS/Pt revealed that the hydrogen production on the Pt site was efficient only when electron donating molecules were used as surface ligands. Based on band gap alignment, the hydrophilic MPA ligands are expected to scavenge holes from $\mathrm{ZnSe}$ but not $\mathrm{ZnTe}$ core NCs. This hypothesis was confirmed by the authors from fluorescence lifetime measurements. Furthermore, the addition of excess ligand molecules to the $\mathrm{NC}$ solution resulted in a recovered $\mathrm{H}_{2}$ generation rates, in agreement with the hypothesized role for the MPA and with the fact that the photo-holes need to be extracted to the $\mathrm{NC}$ surface for the $\mathrm{H}_{2}$ to be produced.

$\mathrm{Cu}_{1.94} \mathrm{~S}-\mathrm{Zn}_{\mathrm{x}} \mathrm{Cd}_{1-\mathrm{x}} \mathrm{S}$ heteronanorods $(0 \leq \mathrm{x} \leq 1)$ have recently been proposed to achieve a tunable composition-dependent band gap (between 2.57- $3.88 \mathrm{eV}$ ) while also favoring charge separation [82]. Even without any cocatalysts, $\mathrm{Cu}_{1.94} \mathrm{~S}-\mathrm{Zn}_{0.23} \mathrm{Cd}_{0.77} \mathrm{~S}$ heteronanorods exhibited an AQE of $8.5 \%$ under visible-light irradiation $(\lambda>420 \mathrm{~nm})$, representing a 59-fold enhancement compared with the pristine $\mathrm{CdS}$ catalyst. Meanwhile, deposition of a Pt cocatalyst on the $\mathrm{Cu}_{1.94} \mathrm{~S}-\mathrm{Zn}_{\mathrm{x}} \mathrm{Cd}_{1-\mathrm{x}} \mathrm{S}$ surface substantially enhanced the hydrogen production performance by achieving an AQE of $26.4 \%$ at $420 \mathrm{~nm}$ [82].

As one example of photocathodes assembled from QDs, Korala et al. have recently reported a new method to fabricate high quality CdSe and CdSe@ZnS QDs photoelectrodes by applying gelation strategies to spin-coated thioglycolic acid-capped QD films (see section 5.1 and figure 9A) [52]. This method enables improved charge transport properties in QD solids by enhancing interparticle coupling via formation of oxidation-induced dichalcogenide linkages. Overcoating CdSe QDs with a thin ZnS shell reduces unpassivated surface states, and thus charge trapping, and results in a more than 2-fold increase of photocurrent $\left(200 \mu \mathrm{A} / \mathrm{cm}^{2}\right.$ versus $75 \mu \mathrm{A} / \mathrm{cm}^{2}$ under white light irradiation and at $-0.57 \mathrm{~V}$ vs $\mathrm{Ag} / \mathrm{AgCl})$.

Cd-free semiconductor NCs.

In the search of alternative light absorber to Cd-based materials, $\mathrm{Cu}_{2} \mathrm{ZnSnS}_{4}$ (CZTS) combines ideal band gap (direct and $1.5 \mathrm{eV}$ ) and a composition based on Earth abundant elements. Yu et al. have constructed HNCs including highly monodisperse quasispherical CZTS as light absorbers and Au and Pt nanoparticles as co-catalysts using the seeded growth approach, with Pt being more active [83]. The best Pt loading in order to maximize the $\mathrm{H}_{2}$ production rate without compromising the light absorption of the CZTS NCs was determined. Furthermore, the compositional tunability allowed the authors to report the $\mathrm{H}_{2}$ production trend versus the composition of the CZTS and observed higher activity in the $\mathrm{Cu}$-poor and $\mathrm{Zn}$-rich samples, in agreement with the behavior observed in photovoltaics [83]. An increased activity was observed when the NCs were functionalized with MPA instead of $\left(\mathrm{NH}_{4}\right)_{2} \mathrm{~S}$. While this behavior is not commented, it highlights once more the importance of controlling interfaces and band alignments in the photocatalytic systems. The same group has also investigated $C u I_{1-x} G a_{x} S_{2}(C I G S)$ NCs 
to identify the best composition to compromise between visible light absorption and efficient charge transfer [84]. In both CIGS and CIGSe, the incorporation of Ga has a major impact on the position of the conduction band minimum, which is shifted to higher energy values; instead, the valence band maximum remains almost unchanged. The additional energy of the electrons at the conduction band minimum when increasing the Ga content, provide extra driving force for charge injection, and thus a faster charge transfer. At the same time though, the band gap increases and so less visible light is absorbed. The optimum composition was found to be $\mathrm{CuIn}_{0.3} \mathrm{Ga}_{0.7} \mathrm{~S}_{2}$, which was characterized by a band gap of $1.75 \mathrm{eV}$. A two-fold increase in the hydrogen production rate was observed when functionalizing the CIGS NCs with Pt.

Among the Cd-free alternative photoanodes for HER, considerable attention has been paid to $\mathrm{CuInS}_{2}$ (CIS) and its alloys; among them an often-studied CIS-based alloy is ZnS$\mathrm{CuInS}_{2}$ (ZCIS) semiconductor [85]. CIS and ZnS are both direct-gap semiconductors with bandgap energies of 1.5 and $3.7 \mathrm{eV}$, respectively. By varying the ratio of CIS to $\mathrm{ZnS}$, the bandgap of ZCIS can be easily tuned to absorb light in the visible region, which makes up a large part of the solar radiation spectrum. Only recently Ye et al. have employed colloidal synthesis to obtain ZCIS nanorods alloy successively decorated with Pt and $\mathrm{Pd}_{4} \mathrm{~S}[86]$. The authors demonstrated the potential utility of these ZCIS NRs in visible light-driven photocatalytic hydrogen generation from water in the presence of $\mathrm{S}^{2-}$ and $\mathrm{SO}_{3}{ }^{2-}$ hole scavengers. The ZCIS-Pt and ZCIS- $\mathrm{Pd}_{4} \mathrm{~S}$ displayed higher photocatalytic performance than the bare ZCIS [86].

Recently Patra et al. have reported the synthesis of 0D and 2D twin dot-disc Au-CIS HNCs (figure 5) [51]. As these structures have plasmon and exciton coupling, they were explored as photocathode in a PEC cell for water splitting and recorded significant enhancement of the photocurrent in comparison to only CIS nanostructures. The half-cell STH evolution efficiency of Au-CIS photocathode was found to be higher $(4.29 \%$ at $0.438 \mathrm{~V}$ vs. RHE) than other reported comparable nanostructures [51]. 

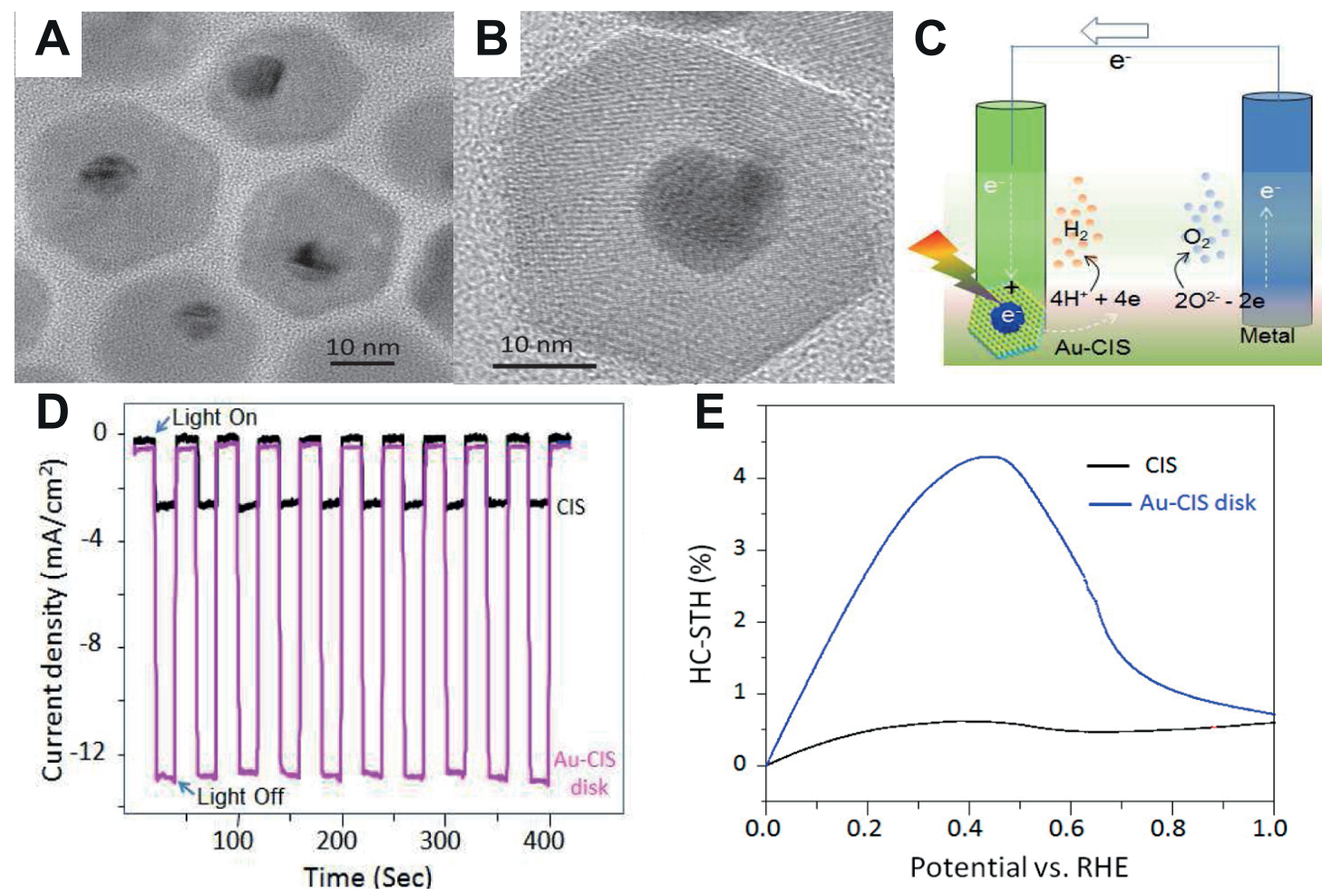

Figure 5. CIS/Au hybrid NCs assembled as photocathodes for HER. (A, B) Low- and high-resolution TEM images of hybrid dot-disk Au-CIS nanostructures. (C) Schematic representation of PEC cell setup (D) Time dependent photocurrent density with illumination switched $\mathrm{ON}$ and OFF for CIS and Au-CIS photocathodes at $-0.5 \mathrm{~V}$ vs SCE[51] (E) Half-cell solar-to-hydrogen evolution (HC-SHE) efficiency of CIS and AuCIS( disk) electrodes. Reprinted with permission from [51], copyright 2016 American Chemical Society

\subsection{Colloidal nanocrystals as light absorbers for OER}

The studies on HER using colloidal NCs largely outnumber the ones on OER. Only a couple of attempts have been made to synthesize colloidal II-VI semiconductor/metal HNCs for OER. However, no oxygen evolution has been demonstrated so far [87]. As discussed in the previous session, the main challenge is the instability of these materials due to photo-oxidation. The latter becomes even more problematic under OER conditions, as the reaction is slower (four holes need to be transferred to liberate one oxygen molecule) and higher overpotentials are usually required. Because of their higher stability in oxidative environment, metal oxide NCs are ideal candidate as light absorbers for OER. A few studies have been reported where photoanode electrodes are assembled from metal oxide NCs. The problem here is that there are not too many stable systems available yet that efficiently absorb visible light. As the previous one, this section is organized by material classes.

II-VI semiconductor NCs.

Kalisman et al. have functionalized $\mathrm{CdSe} @ \mathrm{CdS}$ asymetric dot@rod with $\mathrm{IrO}_{2}$ nanoparticles [87]. While the authors highlight photochemical stability under prolonged illumination, no demonstration of possible utilization of the photogenerated holes is 
reported. More recently a study from the same group details the synthesis of complex hybrid NCs comprising both a water oxidation and water reduction catalyst, namely CdSe@CdS/Pt/Ru@RuxOy [88]. This is a great example of the impressive control gained by the nanoscience community over the assembly of different building blocks in a single object. However, also this work supplies no evidence of actual activity towards water oxidation and/or reduction.

Metal Oxide NCs.

In most of the studies involving colloidal metal oxide NCs, photoanode electrodes are prepared by necking the NCs at a certain temperature. Such a thermal treatment might or might not induce phase transformation and/or grain growth depending on materials and treatment conditions. As illustrated in section 2.1, the facets exposed on the NC surface can be finely tailored by tuning the reaction conditions. One of the advantage of using colloidal NCs as precursors for thin film electrodes is the possibility of directing grain size and orientation, which are known to impact transport properties and catalytic reactivity, through the size and shape of the NCs themselves. Such a tailoring of the microstructure has been demonstrated in materials for photovoltaics and has often lead to enhanced performance [89].

The popular binary metal oxide photoanodes researched upon, like $\mathrm{TiO}_{2}, \mathrm{WO}$, and $\alpha$ $\mathrm{Fe}_{2} \mathrm{O}_{3}$, are readily available by colloidal synthesis.

Recently, our group has been able to position nitrogen dopants selectively in interstitial or substitutional sites in $\mathrm{TiO}_{2}$ branched NCs [90]. Correlating the change of band gap and the photoelectrochemical behavior of the doped $\mathrm{TiO}_{2}$ nanostructures to the nature of the dopants was thus possible. When assembled as photoanodes, the substitutionally doped samples outperformed the interstitially doped ones. While $\mathrm{N}$-doped $\mathrm{TiO}_{2}$ is clearly not a technologically relevant photoanode material, this work demonstrates the potential of colloidal NCs as atomically tunable materials to build unambiguous structure/properties relations, often prevented by a poor angstrom-scale material control.

$\mathrm{WO}_{3}$ photoanode electrodes assembled from nanowires by Goncalves et al or from nanoplates by Hilaire et al have showed preferential texturing along the $\{200\}$ and the $\{002\}$ planes, respectively [91,92]. These photoanodes exhibited a photocurrent of 1.96 $\mathrm{mA} / \mathrm{cm}^{2}$ at $1.23 \mathrm{~V}_{\mathrm{RHE}}$ and $2.7 \mathrm{~mA} / \mathrm{cm}^{2}$ at $1 \mathrm{~V}_{\mathrm{RHE}}$, respectively, both with an onset potential around $0.5 \mathrm{~V}_{\mathrm{RHE}}$. Because of different electrolyte concentration, these results are not directly comparable, yet they serve the purpose of illustrating the impact of texturing on the photocurrents by suggesting a better charge transport along the [001] direction.

Goncalves et al have prepared hematite $\alpha-\mathrm{Fe}_{2} \mathrm{O}_{3}$ photoanodes by annealing at $820^{\circ} \mathrm{C}$ thin films of highly monodisperse magnetite nanocrystals with $2.5 \mathrm{~nm}$ in diameter [93]. Among the crystalline phases of iron oxide, hematite is the active phase to drive water oxidation. The growth of columnar hematite grains along the [001] direction with grain size around $50 \mathrm{~nm}$ was observed. Two main factors were identified to contribute to the lower overpotential and high photocurrent (for 930nm thick films $1.1 \mathrm{~mA} / \mathrm{cm}^{2}$ at $1.23 \mathrm{~V}$ vs RHE and overpotential smaller than $0.8 \mathrm{~V}$ ) recorded for these photoanodes: 1) favorable surface properties of the hematite grains obtained by using a high temperature and an oxygen atmosphere during the sintering process; 2) favorable morphology, where columnar grains are oriented in a favorable crystallographic orientation for electron collection and the mesoporous structure. The same authors achieved better performance (photocurrent up to $1.4 \mathrm{~mA} / \mathrm{cm}^{2}$ and $2.7 \mathrm{~mA} / \mathrm{cm}^{2}$ at $1.23 \mathrm{~V}_{\mathrm{RHE}}$ for undoped and Sn-doped 
hematite films, respectively) when assemblying the colloidal magnetite NCs under an external magnetic field during dip coating deposition [94]. The process is illustrated in figure 6A. Such a process promoted the growth of hematite columnar grains of $180 \mathrm{~nm}$ thickness and with a preferred [110] orientation.

The study by Wang et al on $\mathrm{Sn}$-doped $\mathrm{Fe}_{2} \mathrm{O}_{3}$ corroborates an increased electron transport properties along the same crystallographic direction. The authors reported Sn-doped $\mathrm{Fe}_{2} \mathrm{O}_{3}$ films with a preferred [110] texture, obtained by sintering in Argon Sn-doped magnetite NCs at $550^{\circ} \mathrm{C}$ [95]. A photocurrent up to $3.32 \mathrm{~mA} / \mathrm{cm}^{2}$ at $1.23 \mathrm{~V}$ vs RHE and onset potential around $0.9 \mathrm{~V}$ vs RHE was measured for film thickness of $385 \mathrm{~nm}$. Samples annealed in inert atmospheres performed much better than the ones annealed in air and doped samples better than undoped ones. Sintering in reducing atmosphere promotes the creation of oxygen vacancies which can play a key role in increasing conductivity (which was verified by the authors of this work) as well as modifying the photoactivity towards water oxidation.

In the previous examples, hematite was obtained upon annealing of magnetite NCs. Wang et al. succeeded in synthesizing $\alpha-\mathrm{Fe}_{2} \mathrm{O}_{3} \mathrm{NCs}$ by microwave-assisted colloidal synthesis [96]. Because of the good solubility, the photocatalytic activity was tested in aqueous solution for oxygen evolution. The same particles were deposited by electrophoresis, annealed and tested as thin films electrodes as well. Comparison with other studies is challenging as only oxygen generation rate was reported [96]. However, this work shows the versatility afforded by colloidal chemistry: light absorbing nanocrystals can be tested both as photocatalysts in solution or assembled in photoanode for electrochemical investigation.

In the last years, monoclinic $\mathrm{BiVO}_{4}$ has attracted much attention as a photoanode for solar fuel applications [97-99]. The best performance for this materials have been obtained by nanostructuring and by interfacing with wider band gap oxide semiconductors [99]. Our group has recently developed a $\mathrm{BiVO}_{4} \mathrm{NC}$ ink that facilitates the assembly of $\mathrm{BiVO}_{4} /$ metal oxide $(\mathrm{MO})\left(\mathrm{MO}=\mathrm{TiO}_{2}, \mathrm{WO}_{3}\right.$, and $\left.\mathrm{Al}_{2} \mathrm{O}_{3}\right)$ nanocomposites in which the morphology of the metal oxide building blocks is finely tailored [16]. The $\mathrm{BiVO}_{4} \mathrm{NC}$ ink was constituted by $\mathrm{Bi}_{2} \mathrm{O}_{2.7} / \mathrm{VO}_{\mathrm{x}}$ heterodimers (figure $6 \mathrm{~B}$ ). Tunable nanostructured $\mathrm{BiVO}_{4} / \mathrm{MO}$ were obtained by annealing thin films deposited from mixed solution of the $\mathrm{NC}$ heterodimers with $\mathrm{TiO}_{2}, \mathrm{Al}_{2} \mathrm{O}_{3}$ and $\mathrm{WO}_{3} \mathrm{NC}$ of different size and shape. We used a combination of transient absorption spectroscopy - spanning from picoseconds to second timescales - and photoelectrochemical measurements to reveal that the achieved structural tunability is key to understanding and directing charge separation, transport, and efficiency in these complex oxide heterostructured films. For example, in the $\mathrm{BiVO}_{4} / \mathrm{WO}_{3}$ nanocomposite, better photoelectrochemical performance was found for smaller $\mathrm{WO}_{3} \mathrm{NRs}$ (figure 6B). This behavior resulted from an improved efficiency of electron injection from $\mathrm{BiVO}_{4}$ to $\mathrm{WO}_{3}$ deriving from a more extended interfacial area between $\mathrm{BiVO}_{4}$ and $\mathrm{WO}_{3}$, compared to nanocomposites comprising bigger $\mathrm{WO}_{3}$ nanorods[16]. Our results highlight the significant potential afforded by the assembly of such oxide nanocomposites and unveil relationships between MO properties and PEC performance.

We have also investigated a NC-seed mediated growth of thin films vanadate light absorbers. The seeding process has been traditionally employed to reduce the crystallization temperature in ceramic ferroelectric materials, such a lead zirconate 
titanate, to facilitate their integration in flexible electronics as a result of the decreased crystallization temperature [100,101]. Recently, our group has utilized colloidal nanocrystals with variable composition as seeds for the nucleation and growth of vanadate thin films [102]. Specifically, we employed $\mathrm{Bi}_{x} \mathrm{Sb}_{1-\mathrm{x}}$ colloidal $\mathrm{NCs}$ with variable composition $x$ to discover a new light absorber, $\mathrm{BiV}_{1-\mathrm{x}} \mathrm{Sb}_{\mathrm{x}} \mathrm{O}_{4}$. The $\mathrm{Bi}_{1-\mathrm{x}} \mathrm{Sb}_{\mathrm{x}} \mathrm{NCs}$ were converted into $\mathrm{Sb}-\mathrm{BiVO}_{4}$ films by reacting them with vanadyl acetyacetonate upon annealing (figure 6C). This novel two-steps synthetic approach allowed for a precise compositional tuning while preserving the same morphology and crystalline structure up to a $20 \%$ antimony content. It is important to note that attempts to synthesize $\mathrm{Sb}-\mathrm{BiVO}_{4}$ by more conventional approaches such as sol-gel, solid solution, and spray pyrolysis were unsuccessful and resulted in phase separation, even for low percentage Sb alloys. Thanks to the broad compositional range covered by our approach, a combination of theoretical predication and experimental validation has showed that this photoanode possesses a band gap that linearly decreases with increasing Sb content up to $20 \%$ when the band gap is $0.2 \mathrm{eV}$ smaller than the one of the undoped counterpart (figure $6 \mathrm{C}$ ). While this material is still far from being optimal, the results of this work, derived from close coupling of theoretical understanding and advanced synthesis approaches, provide a path to the design of other metal oxides alloys with desirable band gaps and band edge positions for solar water splitting. To demonstrate the general applicability of the NC-seeded growth approach, other high surface area ternary oxide light absorbers were targeted. $\mathrm{Bi}_{2} \mathrm{WO}_{6}$, $\mathrm{CuWO}_{4}, \mathrm{SnWO}_{6}$, and $\mathrm{InVO}_{4}$ were synthetized by using $\mathrm{Bi}, \mathrm{Cu}, \mathrm{Sn}$ and $\mathrm{In}_{2} \mathrm{O}_{3} \mathrm{NC}$ seeds, respectively. 

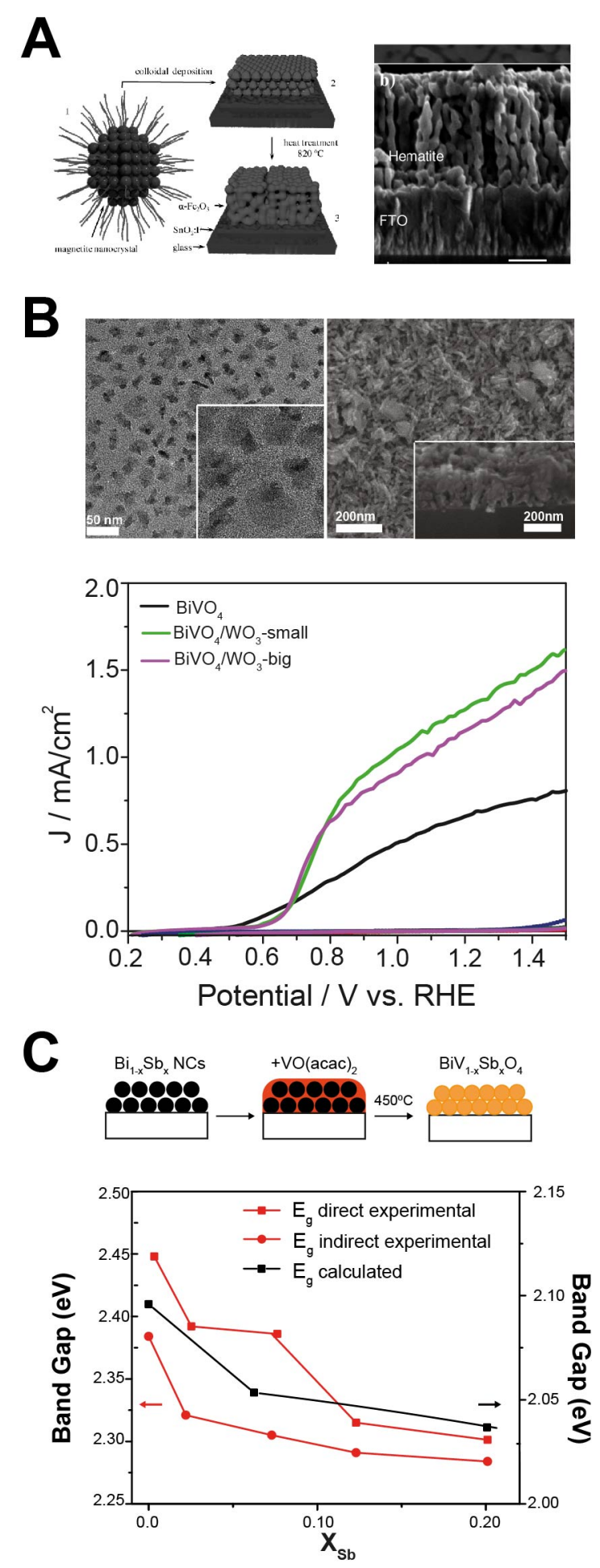

Figure 6. Photoanodes from assembly of metal oxide NCs. (A) Proposed pathway to produce hematite thin-film starting from colloidal magnetic NCs and scanning electron microscopy (SEM) cross section image of the obtained hematite thin film [93]. (B) Lowresolution TEM images of as-synthesized $\mathrm{Bi}_{2} \mathrm{O}_{2.7} / \mathrm{VO}_{x} \mathrm{NC}$ heterodimers (left) and SEM images on the surface and in cross-section (inset) of the $\mathrm{BiVO}_{4} / \mathrm{TiO}_{2}$ nanocomposite 
(right) obtained from assemblying $\mathrm{Bi}_{2} \mathrm{O}_{2.7} / \mathrm{VO}_{x} \mathrm{NC}$ heterodimers with $\mathrm{TiO}_{2} \mathrm{NRs}$; the bottom figure shows the current density vs. potential (J-E) curves of photoelectrodes prepared from the different nanocomposites measured under illumination (continuous traces) and in dark (dashed traces) in a $1 \mathrm{M}$ phosphate buffer $(\mathrm{pH}$ 6.8) containing $0.1 \mathrm{M}$ $\mathrm{Na}_{2} \mathrm{SO}_{3}$ as hole scavenger [16]. (C) Schematic representation for the conversion of $\mathrm{Bi}_{1-x} \mathrm{Sb}_{x} \mathrm{NC}$ seeds to $\mathrm{BiV}_{1-\mathrm{x}} \mathrm{Sb}_{\mathrm{x}} \mathrm{O}_{4}$ films together with the plot of composition-dependent band gap [102]. Reprinted with permission from [16,93], copyright 2015, 2011 American Chemical Society. Reprinted from [102] with permission from John Wiley and Sons, copyright 2015.

\section{Colloidal nanocrystals as electrocatalysts.}

Studies on colloidal NCs as electrocatalysts for HER and OER have proliferated in the last few years and have highlighted the great potential of colloidal NCs both as a platform to deepen the fundamental understanding of structure/properties relations in catalysis and as an actual technological opportunity. The fine tailoring of size, shape and composition serves well the purpose of fundamental studies. The easy processability of colloidal solutions (colloidal NCs can be deposited on flat or porous or temperature sensitive substrates and loading can be easily adjusted) is well suited for integration in different device configurations. Much of the recent work on HER and OER, which is reviewed in this section, focuses on non-noble metal catalysts that are stable in extreme $\mathrm{pH}$ conditions, which are relevant for electrolysis and for integrated photoelectrochemical devices $[103,104]$.

\subsection{Colloidal nanocrystals as electrocatalyst for HER}

Platinum is so far the best catalyst for HER, with the lowest overpotential of $-18 \mathrm{mV}$ at $10 \mathrm{~mA} / \mathrm{cm}^{2}$ and $-26 \mathrm{mV}$ at $-20 \mathrm{~mA} / \mathrm{cm}^{2}$ [103]. Examples of colloidal semiconductor/Pt HNCs for HER have been discussed in section 3.1. Recently the Schaak group has been leading the field by investigating the electrochemical activity of metal phosphide colloidal NCs towards hydrogen evolution and the stability in acidic conditions, under which proton exchange membrane-based electrolysis is operational [105]. An overview of this work is given in figure 7. Before performing any electrocatalytic measurement on these NCs, ligand removal was performed by annealing 30 minutes at $450{ }^{\circ} \mathrm{C}$ in $5 \% \mathrm{H}_{2} / \mathrm{N}_{2}$ without observing any apparent change in the morphology /composition of the particles used in their studies.

Nickel phosphide $\left(\mathrm{Ni}_{2} \mathrm{P}\right)$ NCs were synthesized as hollow multifaceted particles with an average size around $17 \mathrm{~nm}$ and high density (001) facets exposed, which has previously been predicted to be the most active surfaces [106]. The overpotential for these particles to produce current densities of -20 and $-100 \mathrm{~mA} / \mathrm{cm}^{2}$ were $-130 \mathrm{mV}$ and $-180 \mathrm{mV}$, respectively. The size and shape-dependence of electrocatalytic activity of the $\mathrm{Ni}_{2} \mathrm{P} \mathrm{NCs}$ has also been investigated [107]. Electrochemical measurements have revealed that $\mathrm{Ni}_{2} \mathrm{P}$ nanospheres $\left(\mathrm{Ni}_{2} \mathrm{P} \mathrm{NSs}\right)$ with predominant (001) surfaces exhibit higher HER activity than $\mathrm{Ni}_{2} \mathrm{P}$ nanorods $\left(\mathrm{Ni}_{2} \mathrm{P}\right.$ NRs) with the (210) surface. $\mathrm{Ni}_{2} \mathrm{P}$ NSs generated a current density of $-10 \mathrm{~mA} \mathrm{~cm}^{-2}$ at an overpotential of $135 \mathrm{mV}$ in $0.5 \mathrm{M} \mathrm{H}_{2} \mathrm{SO}_{4}$, whereas $\mathrm{Ni}_{2} \mathrm{P}$ NRs produced the same current density at a larger overpotential $(270 \mathrm{mV})$. Furthermore, the turnover frequency of $\mathrm{Ni}_{2} \mathrm{P}$ NSs was about thirteen times higher than that of $\mathrm{Ni}_{2} \mathrm{P}$ 
NRs. The results suggest that the crystallographic facets of $\mathrm{Ni}_{2} \mathrm{P}$ NCs play a critical role in dictating HER activities [107]. Zhou et al. have also investigated the effect of the size of $\mathrm{Ni}_{2} \mathrm{P}$ NCs for HER to establish the relationship between size and catalytic activity [108]. Highly monodisperse, small-sized $\mathrm{Ni}_{2} \mathrm{P}$ NCs with sizes ranging from $2 \mathrm{~nm}$ to 10 $\mathrm{nm}$ were synthesized. The resulting small-sized product possesses a high accessible surface area and a high density of exposed (001) facets. Experimental results showed that the small-sized $\mathrm{Ni}_{2} \mathrm{P}$ NCs not only reduced the overpotential but also decreased the Tafel slope, thus significantly improving HER catalytic performance. The best performance were achieved with $5.4 \mathrm{~nm} \mathrm{Ni}_{2} \mathrm{P}$ NCs which showed an overpotential of $93 \mathrm{mV}$ at -20 $\mathrm{mA} / \mathrm{cm}^{2}$ and for a mass loading of approximately $1 \mathrm{mg} / \mathrm{cm}^{2}$ onto a $0.20 \mathrm{~cm}^{2}$ Ti foil substrate [108].

Multi-faceted cobalt phosphide (CoP) NCs were synthesized by the Schaak group by reacting Co NCs with trioctylphosphine [109]. Electrodes comprised of CoP NCs on a Ti support (mass loading equal to $2 \mathrm{mg} / \mathrm{cm}^{2}$ ) produced a cathodic current density of 20 $\mathrm{mA} / \mathrm{cm}^{2}$ at an overpotential of $-85 \mathrm{mV}$. The CoP/Ti electrodes were stable over $24 \mathrm{~h}$ of sustained hydrogen production in $0.50 \mathrm{M} \mathrm{H}_{2} \mathrm{SO}_{4}$ [109]. The same authors used the tunability afforded by colloidal chemistry to directly compare the activity of CoP and $\mathrm{Co}_{2} \mathrm{P} \mathrm{NC}$ catalysts [110]. $\mathrm{Co}_{2} \mathrm{P}$ has not been studied as extensively as CoP because of its higher overpotentials. However, unambiguous conclusions from comparing catalysts synthesized by different approaches and tested under different conditions cannot be made. Nevertheless, such comparisons are needed to identify and to understand the key intrinsic factors that underpin high catalytic activity. Morphologically equivalent $\mathrm{CoP}$ and $\mathrm{Co}_{2} \mathrm{P}$ multifaceted and hollow particles were accessed using the same colloidal procedure. This allows similarities and differences in HER activity to be correlated most significantly with composition and structure, while minimizing morphological contributions, such as size, size distribution, dispersability, and faceting. When testing the electrocatalytic activity of $\mathrm{CoP}$ and $\mathrm{Co}_{2} \mathrm{P} \mathrm{NCs}$, the following activity trend was found $\mathrm{Co}<\mathrm{Co}_{2} \mathrm{P}<\mathrm{CoP}$. While this result is in agreement with previous literature, much lower overpotential $(-95 \mathrm{mV}$ and $-109 \mathrm{mV}$ to produce cathodic current densities of -10 and -20 $\mathrm{mA} / \mathrm{cm}^{2}$, respectively) was found for the $\mathrm{Co}_{2} \mathrm{P}$ NCs compared to the ones with different morphology reported in the literature (that falls in a range between $-160 \mathrm{mV}$ and -200 $\mathrm{mV}$ at $-20 \mathrm{~mA} / \mathrm{cm}^{2}$ ), once again it reveals the importance of morphology in catalysis. Furthermore, direct comparisons by the same group of multifaceted $\mathrm{CoP}$ nanoparticles with highly branched CoP nanostructures that exposed a high density of (111) facets suggested that the high HER activity of $\mathrm{CoP}$ is intrinsic to the system and that, for this system, shape may not play a significant role in defining the magnitude of the overpotentials required to produce operationally relevant cathodic current densities [111]. Ha et al. have investigated the active site and stability of the same colloidally synthesized CoP NCs for HER trough experiments and DFT calculations [112]. The HER activity of $\mathrm{CoP}$ is found to be reduced with potentiostatic holding at potentials above $0.4 \mathrm{~V}$ vs. RHE. This activity loss was accompanied by leaching of $\mathrm{P}$ and lowered $\mathrm{Co} / \mathrm{P}$ ratio suggested by EDX results. The formation of (oxy)phosphate(s) emerges concomitant with activity reduction as indicated by signal changes of $\mathrm{Co}$ and $\mathrm{P}$ in X-Ray absorption spectroscopies. Furthermore, DFT calculations indicate surface $P$ could act as the active site for HER on CoP, which constrasts with the traditional HER mechanisms on the metal site [112]. Iron phosphide (FeP) NCs were synthesized and tested by the Schaak's group [113]. 
These particles possess a similar morphology (hollow 10nm spheres) compared to the nickel and cobalt phosphide, though they outperformed those. In fact, they showed an overpotential of $-50 \mathrm{mV}$ under the same acidic conditions the other transition metal phosphides had been tested, and $-102 \mathrm{mV}$ in a neutral electrolyte, at a current density of $10 \mathrm{~mA} / \mathrm{cm}^{2}$. In addition to serving as an efficient HER electrocatalyst, FeP nanoparticles were immobilized on $\mathrm{TiO}_{2}$ particles (Degussa P25) to photocatalytically generate hydrogen under UV illumination in acidic and neutral-pH aqueous solutions.

Amorphous tungsten phosphide (WP) nanoparticles have been synthesized and demonstrated to be highly active HER electrocatalysts in acidic solutions, requiring overpotentials as low as $-120 \mathrm{mV}$ and $-140 \mathrm{mV}$ at current densities of $-10 \mathrm{~mA} / \mathrm{cm}^{2}$ and $20 \mathrm{~mA} / \mathrm{cm}^{2}$, respectively [114].

Finally, the Schaak group has also demonstrated that colloidal molybdenum phosphide (MoP) amorphous NCs with diameter around $4 \mathrm{~nm}$ are active and acid-stable electrocatalysts for HER [115]. Overpotential as low as -90 and $-105 \mathrm{mV}$ were requested to produce operationally relevant current densities of -10 and $-20 \mathrm{~mA} / \mathrm{cm}^{2}$, respectively, thus revealing that these particles are much more active than bulkier MoP nanostructured electrode ranging between -125 and $-150 \mathrm{mV}$ at $-10 \mathrm{~mA} / \mathrm{cm}^{2}$.
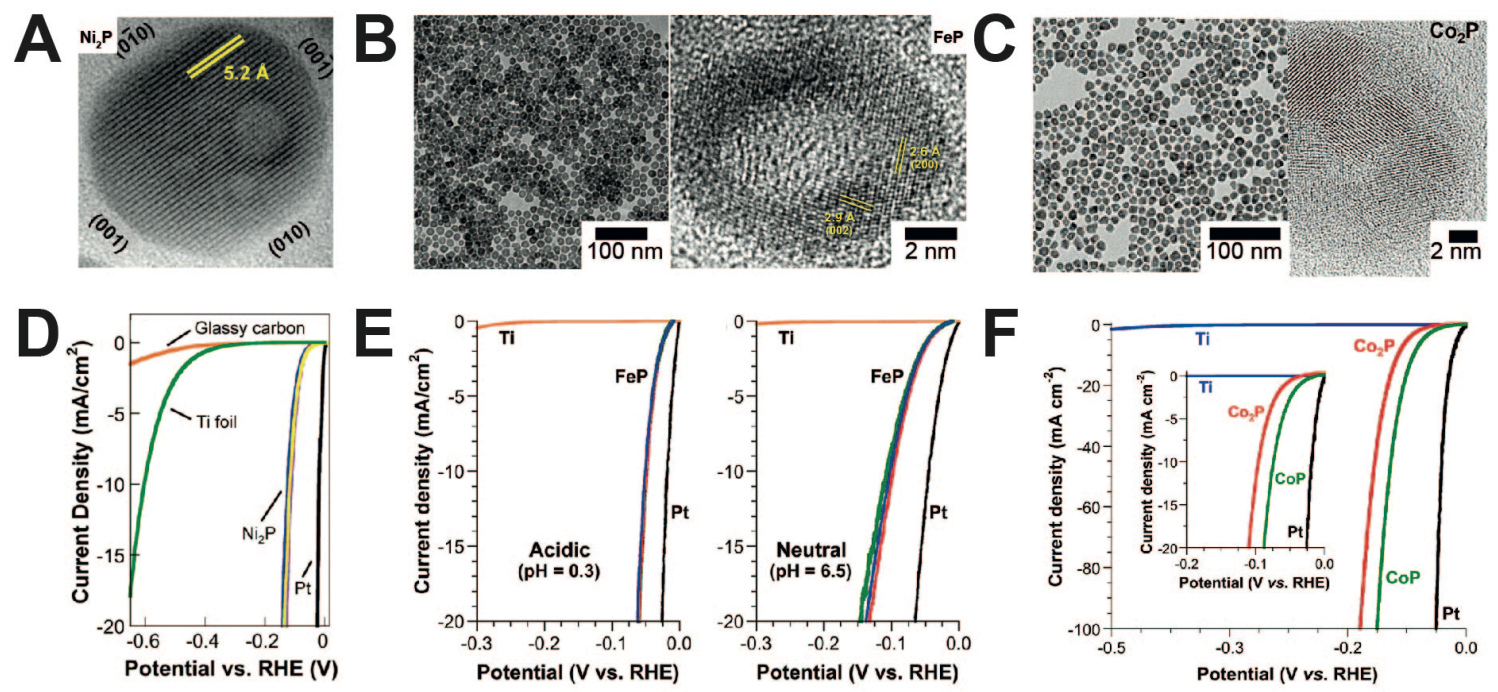

Figure 7. Metal phosphide NCs as electrocatalysts for HER: (A) High resolution (HR)TEM image of $\mathrm{Ni}_{2} \mathrm{P}$ NCs, highlighting the exposed $\mathrm{Ni}_{2} \mathrm{P}(001)$ facet and the 5.2 A lattice fringes that correspond to (010) planes [106]. (B) Low and HR-TEM image of FeP NCs [113]. (C) Low and HR-TEM image of $\mathrm{Co}_{2} \mathrm{P}$ NCs[110]. (D) Plot of current data vs. potential in $0.5 \mathrm{M} \mathrm{H}_{2} \mathrm{SO}_{4}$ for $\mathrm{Ni}_{2} \mathrm{P} / \mathrm{Ti}$ electrodes [106]. (E) Plot of current data vs. potential for three distinct $\mathrm{FeP} / \mathrm{Ti}$ electrodes along with $\mathrm{Pt}$ and $\mathrm{Ti}$ in (acidic) $0.5 \mathrm{M} \mathrm{H}_{2} \mathrm{SO}_{4}$ as well as in (neutral) 1.0 M Phosphate buffered saline (PBS) [113]. (F) Plot of current data vs. potential in $0.5 \mathrm{M} \mathrm{H}_{2} \mathrm{SO}_{4}$ for $\mathrm{Co}_{2} \mathrm{P} / \mathrm{Ti}$ and $\mathrm{CoP} / \mathrm{Ti}$ electrodes [110]. Reprinted with permission from [106,110,113], copyright 2013, 2015, 2014 American Chemical Society.

All the previous catalysts have been tested in acidic conditions. However, finding HER catalysts which are active and stable in alkaline solution is highly sought after as well. 
Recently, the Schaak group has reported the colloidal synthesis of molybdenum rich $\mathrm{Co}$ Mo alloy NCs with diameters of approx. $3 \mathrm{~nm}$ and they have proved their potential to serve as an Earth abundant alternative to Pt for catalyzing the HER in alkaline aqueous solutions [116]. With an observed overpotential of $-75 \mathrm{mV}$ at an operationally relevant current density of $-10 \mathrm{~mA} / \mathrm{cm}^{2}$, the performance of the Co-Mo/Ti electrode places it amongst the best non-noble metal HER catalysts in alkaline solutions. When subjected to both short-term galvanostatic stability testing and longer-term simulated stability testing via cyclic voltammetry, the $\mathrm{Co}-\mathrm{Mo} / \mathrm{Ti}$ electrode was found to be stable in $1 \mathrm{M} \mathrm{KOH}$ [116].

While none of the catalysts reviewed above outperform $\mathrm{Pt}$, these studies demonstrate that phosphide NCs still represent valid non-noble metal alternative electrocatalyst.

\subsection{Colloidal nanocrystals as electrocatalyst for OER}

As referred to in section 3.2, OER is more challenging than HER because of the slower kinetics of the $4 \mathrm{e}^{-} / 4 \mathrm{H}^{+}$coupled transfer needed to liberate one oxygen molecule. The search towards robust Earth abundant electrocatalysts is frenetic but simultaneously extremely challenging. One noteworthy observation is that iridium and ruthenium are the only OER catalysts active and stable in acidic electrolytes. Surprisingly not many studies on ruthenium and iridium oxide colloidal NCs, prepared in organic solvents, to investigate size and shape dependence have been reported. Under alkaline conditions, a few more options exist, with NiFe-based catalysts being the most investigated so far. To the best of our knowledge only a handful of studies have been reported of OER catalysts prepared by a colloidal approach.

Work from the group led by Bein and Fattakhova-Rohlfing focuses on the synthesis and characterization of $\mathrm{NiO}$ and $\mathrm{Fe}$-doped $\mathrm{NiO} \mathrm{NCs}$ (figure 8) [117]. Those were prepared by solvo-thermal synthesis in tert-butanol and characterized by a size below $5 \mathrm{~nm}$ with a slight decrease in size as the iron content increases up to $20 \%$. It is interesting to note that, unlike the previously reported ultrathin $\mathrm{NiO}$ films, NiO NCs do not completely transform into layered $\mathrm{Ni}(\mathrm{OH})_{2}$ after prolonged cycling. This behavior is not discussed further by the authors but it is a clear indication that that catalytic mechanism might be different in nanoscale catalysts. While there are no big changes in overpotenital compared to bulk, the beneficial effect of the reduced crystal size of undoped $\mathrm{NiO}$ to only a few nanometers and the surface properties of the nanoparticles on the catalytic performance is shown by the very high turnover frequency value $\left(0.55 \mathrm{~s}^{-1}\right)$, indicating an increased amount of electrocatalytically active sites. As for the Fe-doped $\mathrm{NiO}$, while different studies report a solubility limit up to 5-6\% before phase separation occurs, the authors did not observe any formation of additional phases up to $20 \%$ according to the results of several characterization methods. The unusually high solubility of Fe in the rock salt $\mathrm{NiO}$ structure can originate from the kinetic control of the $\mathrm{Fe}-\mathrm{NiO}$ phase formation in a solvothermal process, as well as the effect of the nanoscale where the metastable and defect phases often show higher stability than in the bulk. The NC dispersion was deposited as thin films on gold electrodes of the piezoelectric quartz crystals for exact determination of their loading. When doping the nanocrystals with iron, the catalytic activity of the $\mathrm{NiO}$ was even further increased with turnover frequency up to $1.9 \mathrm{~s}^{-1}$ for $10 \% \mathrm{Fe}$ nanoparticles in the present study. In addition to the extremely high electrocatalytic activity, the excellent dispersibility of the $\mathrm{Fe}_{\mathrm{x}} \mathrm{Ni}_{1-\mathrm{x}} \mathrm{O}$ nanoparticles offers 
additional advantages, such as a great flexibility towards the fabrication of different electrode architectures.

Bau et al. have synthesized and tested as OER electrocatalysts $8 \mathrm{~nm} \mathrm{Ni-Fe-O} \mathrm{NCs} \mathrm{in} \mathrm{a}$ metastable rock-salt phase and variable composition ratios of nickel to iron $[118,119]$. The nickel-rich NCs showed the lowest overpotential of around $300 \mathrm{mV}$ at $10 \mathrm{~mA} / \mathrm{cm}^{2}$ with optimal electrode thickness corresponding to a couple of monolayers. The similar OER performance of the rock salt phase NCs with other Ni-Fe-O based catalysts suggests that the presence of $\mathrm{Fe}^{3+}$ centers on the surface plays a bigger role than the crystalline phase itself.
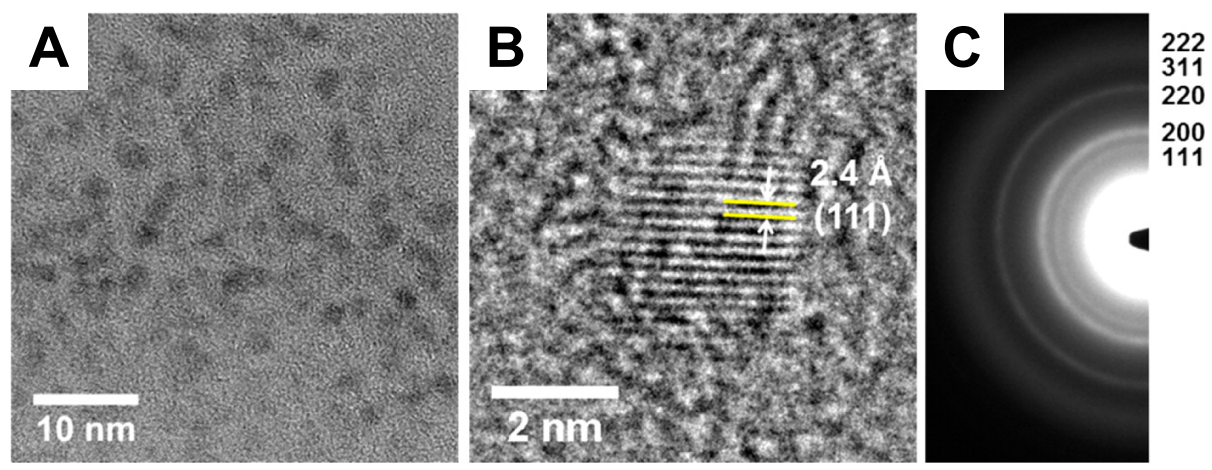

Potential - iR / V vs. Ag/AgCl

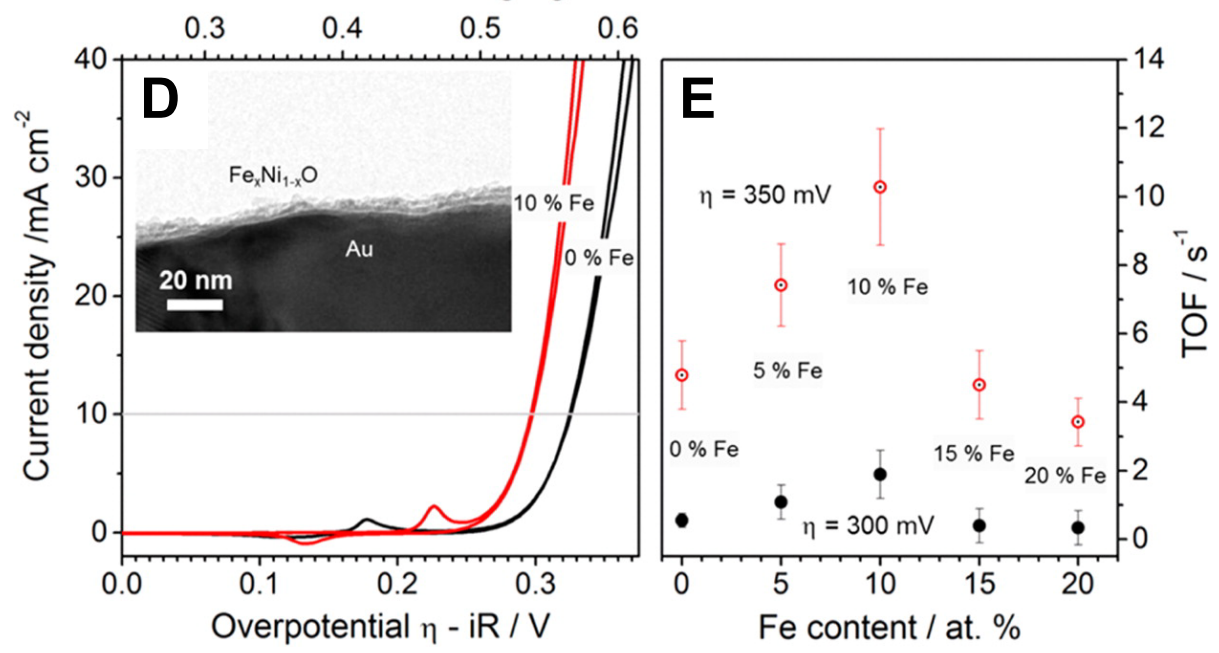

Figure 8. Fe-doped $\mathrm{NiO} \mathrm{NCs}$ as OER electrocatalysts. (A-C) TEM, HRTEM and electron diffraction pattern of $\mathrm{Fe}_{0.1} \mathrm{Ni}_{0.9} \mathrm{O} \mathrm{NCs}$; (D) Cyclic voltammograms of gold electrodes coated with thin films of the NCs with different Fe content. The inset shows across-section HR-TEM image of the NP-10\% film deposited by spin-coating on the substrate. (E) TOF values at an overpotential of $\eta=300 \mathrm{mV}$ (filled circles) and $\eta=350$ $\mathrm{mV}$ (open circles) for different Fe contents [117]. Reprinted with permission from [117], copyright 2015 American Chemical Society. 
In addition to metal oxides, metal phosphides have recently emerged as potential electrocatalyst candidates for OER. Masud et al. have tested colloidal 5nm $\mathrm{FeP}$ NCs and measured an overpotential of $290 \mathrm{mV}$ at a current density of $10 \mathrm{~mA} / \mathrm{cm}^{2}$ [120]. The electrode was reported stable with full activity for 4 hours. In addition, a hybrid electrode assembled from the same NCs and reduced Graphene oxide (rGO) lowered the overpotential to $260 \mathrm{mV}$ at $10 \mathrm{~mA} / \mathrm{cm}^{2}$, making it the lowest reported at the time for metal phosphides. The enhanced electroactivity in the nanocomposites was attributed to the increase in the catalytically active surface area. Dutta et al have studied 1D needle shaped $\mathrm{Co}_{2} P[121]$. These catalysts generated $10 \mathrm{~mA} / \mathrm{cm}^{2}$ at an overpotential of $310 \mathrm{mV}$ and were stable for 10 hours with $15 \mathrm{mV}$ increase in overpotential. The salient feature of this colloidal synthesis is the needle shaped $\mathrm{Co}_{2} \mathrm{P}$, which has higher metallic content compared to $\mathrm{CoP}$ and thereby a most likely better charge transfer. Another interesting result discussed by the authors is the analysis of the electrode after catalysis. A combination of EDX and XPS evidences the presence of oxide or oxo-hydroxide layer around the $\mathrm{Co}_{2} \mathrm{P}$ core. The authors suggest that the $\mathrm{Co}_{2} \mathrm{P} /$ oxide interface enhances the carrier transportation from the core to the oxide layer, thereby enhancing the electroactivity. NiP nanowires were prepared by colloidal synthesis and reported to exhibit current density of $10 \mathrm{~mA} / \mathrm{cm}^{2}$ at an overpotential of $330 \mathrm{mV}$ for OER [122]. As for cobalt phosphide, the authors report the formation of $\mathrm{NiO}_{\mathrm{x}}$ shell during operation which enhances the activity of the electrode for OER and is termed as a synergistic effect which needs further study. The insertion of a second metal has been suggested to lower the thermodynamic barrier of a proton-coupled electron transfer pre-equilibrium while facilitating $\mathrm{O}-\mathrm{O}$ bond formation, leading to enhanced catalytic activity [123]. Hence CoMnP NCs have been synthesized by colloidal synthesis [123]. An overpotential of 330 $\mathrm{mV}$ at $10 \mathrm{~mA} / \mathrm{cm}^{2}$ was reported, which was lower than the overpotential of $\mathrm{CoMnO}_{2}(390$ $\mathrm{mV}$ ) synthesized by the same approach. The higher intrinsic conductivity of phosphides in comparison to oxide has been hypothesized to account for this behaviour. An increase in overpotential to $370 \mathrm{mV}$ on 500 cyclic voltammetry sweeps was observed and attributed to surface oxidation and leaching of $\mathrm{P}$ into the electrolyte. From these initial studies on phosphides as OER catalysts, it is clear that transformation and oxidation of the catalysts occur during operation.

Overall, the studies reviewed in this section also highlights that the minimum overpotential reached for OER with any non-noble metal catalyst tested so far is around $300 \mathrm{mV}$ at a current density of $10 \mathrm{~mA} / \mathrm{cm}^{2}$. Carefully in-situ and in-operando studies are mandatory to get true insights into the activity and reaction mechanisms, so to guide the development of new and more efficient OER catalysts.

\section{Outlook}

\subsection{Ligands off or on?}

As described in section 2, the organic ligands functionalizing the NC surface are essential during the synthesis to modulate NC size and shape and, afterwards, to grant solubility to the NCs in a wide range of solvents. However, they are not necessarily beneficial for their final application [124,125]. In general ligands are hydrophobic and highly insulating, and constitute a significant barrier for charge transfer and transport. Thus, whether the photocatalytic, photoelectrocatalytic or electrocatalytic activity of the NCs is tested in thin films or in solution, proper surface chemistry is needed to investigate their 
behavior with minimal interference of the native coordinating ligands. Fortunately, the understanding and the progress made in NC surface chemistry are far enough that this does not constitute any obstacle for the use of colloidal NCs as model catalytic systems for different reactions [26-30]. The goal is to eliminate/exchange the bulky organic ligands while preserving size, shape and composition of the NCs. Different options are available, with the best one being highly dependent on the system of interest and on whether the NCs will be tested as thin film electrodes or dispersed in solution.

When the NCs are assembled into photoelectrodes or electrodes, thermal treatment to decompose the ligands is one of the most straightforward options. The temperature required for the decomposition of long-chain ligands usually ranges between $400^{\circ} \mathrm{C}$ and $500^{\circ} \mathrm{C}$. Annealing in air is required to avoid carbon contamination from decomposed ligands, which is a problem for light absorption in the case for photoelectrodes. Clearly, this treatment is limited to systems that are resistant to oxidation. We have found such approach to work well the $\mathrm{BiVO}_{4} \mathrm{NC}$ ink and its nanocomposites (figure 6B) [16]. Nickel iron oxide NCs for OER were also annealed in air (figure 8) [117,119]. The Schaak group uses annealing in a tube furnace at a temperature between $350^{\circ} \mathrm{C}$ and $450^{\circ} \mathrm{C}$ under $\mathrm{H}_{2}(5 \%) / \operatorname{Ar}(95 \%)$ to remove surfactant molecules from the surface of their phosphide NCs (figure 6) [106,111,114-116]. For certain NC compositions, at these relatively high temperatures, sintering might occur, thus invalidating the synthetic efforts to control size and shape. One possibility to overcome this issue is to exchange of the native ligands with shorter chain ligands. This approach brings two advantages: i) decomposition temperature is reduced to $<200^{\circ} \mathrm{C}$; ii) annealing under inert atmosphere with minimal carbon residue remaining is possible. Formic acid has worked well for assemblying conductive electrodes from tin-doped indium oxide NCs [126]. Patra et al have used a similar approach when building photoelectrodes from CIS-Au hybrid NCs (figure 4) ${ }^{48}$. Substitution of oleylamine with 1,2-ethane dithiol decreased the annealing temperature to $200^{\circ} \mathrm{C}$ and allowed the ligand removal to be conducted in vacuum instead of air [51]. Alternative exchange approaches have been developed during the last years and include a variety of agents that react with the native NC ligands and leave behind a "bare" NCs surface ("reactive stripping"). The nitrosyl tetrafluoroborate and the exceptionally mild Meerwein's salt are two example among these [127,128]. We have found the latter to be suitable for $\mathrm{N}-\mathrm{TiO}_{2}$ as it allowed annealing under inert atmosphere, which was essential to preserve the nitrogen doping [90]. To build photoelectrodes from $\mathrm{CdSe}$ and CdSe@ZnS core@shell, Korala et al created an interconnected NC network by interparticle linking via dichalcogenide bonding (figure 9A) [52]. Such a process was achieved by exchanging the original trioctylphosphine/stearic acid-capped NCs with thiolated ligands (thioglycolic acid) and inducing gelation afterwards by immersing into an oxidizing solution. This last step was followed by annealing at $250^{\circ} \mathrm{C}$ under argon to form the inorganic sulfur bond between the NCs and an electrically conductive films where size, shape and optical properties of the original NCs were preserved. Fully inorganic ligand, such as molecular metal chalcogenides and halide ligands, have been used to exchange the native ligands and imprint electrical conductivity to NC films $[129,130]$. Yet, these approaches have not been employed to build photoelectrodes for artificial photosynthesis. Very recently, rapid thermal annealing at $750^{\circ} \mathrm{C}$ for 30 seconds has been suggested as method to efficiently remove the ligands from Pd NCs supported on alumina or on ceria without causing any change in the size, shape and composition of 
these NCs [27]. However, its applicability to other system has to be proven yet. Oxygen plasma or UV treatments are other possibilities [119,131]. Bau et al have investigated these two different treatments for ligand removal from the surface of Ni-Fe-O NCs for OER: UV irradiation and air plasma (figure 9B) [119]. Interestingly, only the electrodes treated by uv irradiation were stable and preserved their appearence after electrolysis. The authors claim an increased adherence with the conductive substrate [119].

When the NCs are tested in solution, the need to preserve colloidal stability in water demands for the substitution of the native hydrophobic ligands with hydrophilic ligands. Some of the most common ligand that have been used for this purpose are mercaptoundecanoic acid (MUA), mercaptopropionic acid (MPA), cysteine, citric acid. It has been noted that these ligands themselves can act as holes or electrons scavenger depending on the alignment between the energetic level of the NCs and the ligand. One example is given by Acharya et al and it is illustrated in figure 4C [80]. In a second example, Wang et al have studied the effect of three different ligands on the photocatalytic activity of $\mathrm{H}_{2}$ generation for CdSe@CdS core@shell NCs [132]. They functionalized the NCs with three ligands containing thiol groups: poly-acrylic acid (PAA), 3-mercaptopropionic acid (3-MPA) and 2,3-dimercaptosuccinic acid (DMSA). The DMSA-capped QDs exhibited the highest efficiency of $\mathrm{H}_{2}$ production, while the 3MPA and PAA coatings resulted in the intermediate and lowest photocatalytic activity, respectively. A combination of photoluminescence intensity, lifetime measurements and electro transfer studies illustrated that such a ligand-dependent photocatalytic activity could be mainly attributed to the variations in the surface traps and corresponding charge separation efficiencies of the QDs induced by surface coating with the different ligands [132]. In one last example, Han et al have reported about a robust and highly active system for HER where the dihydropolic acid (DHLA) capping CdSe NCs plays a central role in transferring electrons to the catalysts, which is a soluble $\mathrm{Ni}^{2+}$-DHLA complex [48]. A schematic representation is shown in figure 9C. This system achieved QY of over $36 \%$ and 360 hours stability under illumination at $520 \mathrm{~nm}$ [48].

As discussed already in section 3.1, strategies to stabilize QDs need to be developed for these systems to be considered in a real-world context. Hole accepting ligands with tunable energetic levels can be utilized to conduct detailed charge transfer studies $[72,133]$. Strategies to improve the stability of quantum dots could emerge as a result of a more fundamental understanding of hole transfer. In a recent work, hole transfer kinetic studies have been conducted on CdSe@CdS core@shell NCs to hole acceptors covalently linked to the nanocrystals via a thiolate binding group $[72,133]$. The hole acceptor used is a ferrocene molecule that is positioned at the end of the thiol chain. The large thermodynamic driving force for photoinduced hole transfer allows this process to compete with native recombination. Yet, the hole transfer rate was still extremely sensitive to modulation in the electronic coupling achieved by varying the thickness and composition of the barrier material between the CdSe core and the acceptor. This barrier material includes both the length of aliphatic thiol chain and the thickness of the CdS shell. Through this study the authors demonstrate that one can use the design of QD heterostructures and acceptor ligands to mitigate undesirable trap-states while still being able to extract charges and preserve the photostability.

Recently, De Roo et alhave introduced the concept of sustained chemically driven ligand displacement which allows to address the activity/stability tradeoff in nanocatalysis 
[125]. Herein, weakly binding amines and alcohols chemically convert carboxylic acids functionalizing the surface of $\mathrm{HfO}_{2} \mathrm{NCs}$ into non-coordinate amides and esthers. Thus, the carboxylic acids act both as stabilizer and as reagents and are supplied back during the catalytic cycles [125]. Eventually, similar concepts might also be applied to photocatalysts and catalysts for water splitting.

Overall, these last examples point to the importance of ligand engineering in catalysis, which has been recently gaining increasing attention $[134,135]$. The ligands could indeed play the role of co-catalysts and synergistically contribute to increase the activity or the stability of the catalysts, instead of being always demonized.

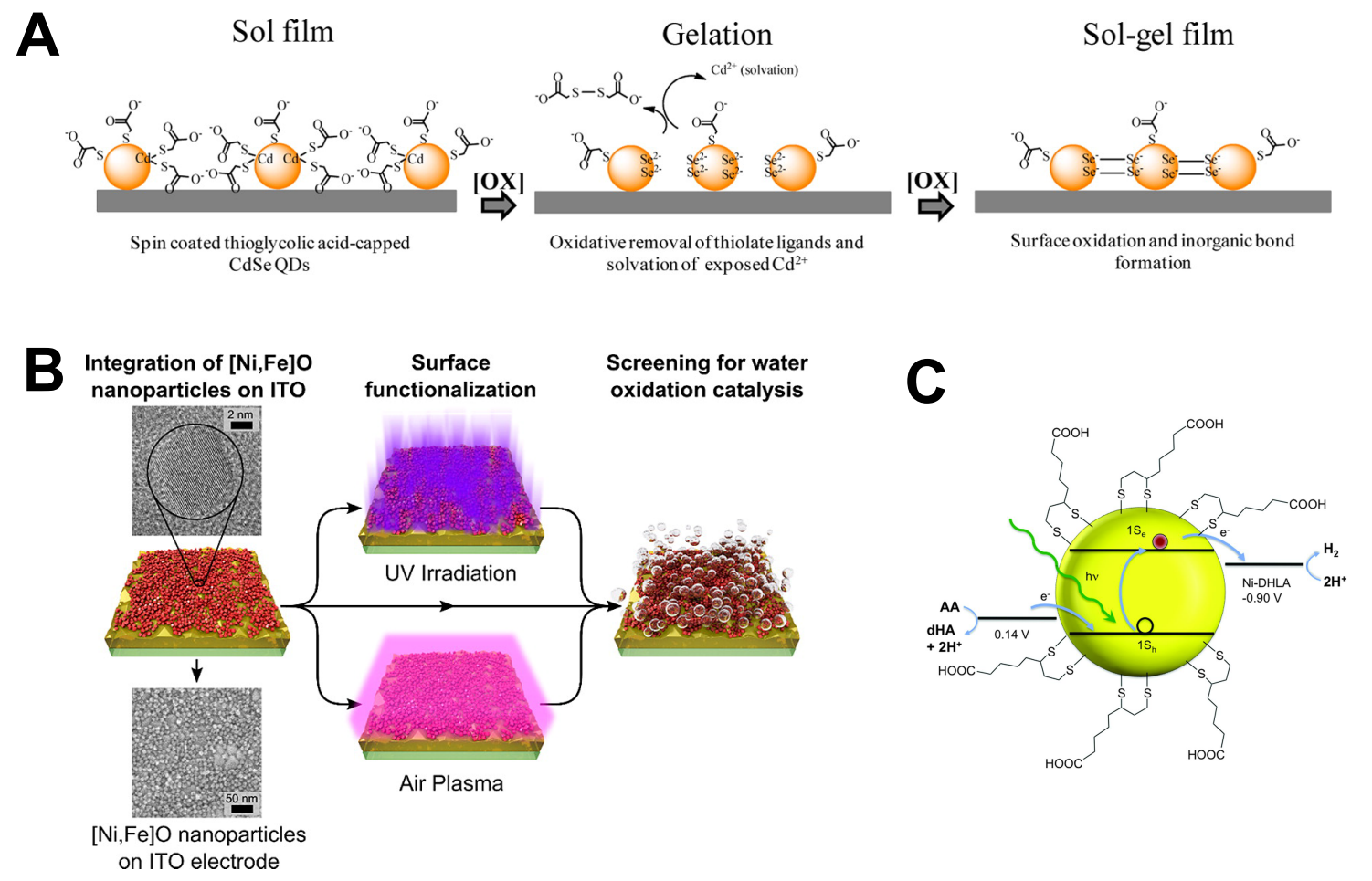

Figure 9. Various strategies for ligand exchange or removal. (A) Schematic representation of the mechanism of oxidative ([OX]) gelation of a thioglycolic acidcapped QD film (sol film) submerged in a tetranitromethane (TNM) solution [52]. (B) Sketch of the functionalization of electrodes with $[\mathrm{Ni}, \mathrm{Fe}] \mathrm{O}$ NCs and subsequent removal of oleate ligands with UV irradiation or air plasma [119]. (C) Cartoon that illustrates the CdSe nanocrystals capped with DHLA with the relevant energies for $\mathrm{H}_{2}$ production. dHA indicates dehydroascorbic acid. Potentials are shown versus that of an NHE at $\mathrm{pH}=$ 4.5 [48]. Reprinted with permission from [52,119], Copyright 2013,2015 American Chemical Society. From [48], reprinted with permission from AAAS.

\subsection{Colloidal chemistry for material discovery}

As evidenced in the previous sections, colloidal chemistry is a powerful synthetic approach to access homo-materials and hetero-materials with a precise control on morphology and composition. One aspect that could be further explored is the use of colloidal approaches for material discovery. New light absorbers and electrocatalysts are required, with a more urgent need in OER compared to HER.

Our group has recently started to explore the potential of using NC-seeded growth to 
synthesize a new light absorber, $\mathrm{Sb}-\mathrm{BiVO}_{4}$ in a wide compositional range (figure 6C) [102]. This same approach could be applied to other systems. More efforts should also be directed towards the colloidal synthesis of these materials. Compared to the classical IIVI QDs and to multinary chalcogenide NCs, the chemistry of multinary metal oxides has not been fully elucidated yet. Also, the complexity of their phase diagrams makes not trivial to isolate them in one single crystalline phase. Often amorphous NCs are obtained. Our work on $\mathrm{BiVO}_{4} \mathrm{NC}$ ink is one example of the challenges related to the synthesis of colloidal visible light absorbing metal oxides [16]. While targeting $\mathrm{BiVO}_{4} \mathrm{NCs}$, we obtained only phase segregated $\mathrm{Bi}_{2} \mathrm{O}_{2.7} / \mathrm{VO}_{x} \mathrm{NC}$ heterodimers, indicating that the chemistry of these vanadates is far from being fully understood. At the same time, we have also demonstrated the power of a rational synthetic design to position dopants in specific lattice sites, for the case of the well-studied $\mathrm{N}-\mathrm{TiO}_{2} \mathrm{NCs}$, and to study their impact on optoelectronic properties and photoelectrochemical performance [37,90]. In addition to enable fundamental studies, overcoming the synthetic challenges to access multinary metal oxide colloidal NCs might open new opportunities towards their actual implementation into baggie-type reactors, which will be discussed in the next section.

Another advantage of colloidal chemistry, being an out-of-equilibrium synthetic approach, is the wider compositional space which can be covered compared to other thermodynamic-governed method. One example cited above concerns Ni-Fe-O OER catalysts but many more could be mentioned [117].

As for the electrocatalyst, non-noble metal catalysts with low overpotential for OER have yet to be found. The situation is even more dramatic in terms of overpotential and selectivity if one starts to look at the $\mathrm{CO}_{2}$ reduction reaction, which is out of the scope of the present review. At the nanoscale property modification can be tremendous because of the unique effects, such as the variation of the electronic state density, the charge redistribution, surface- and strain-driven lattice distortion, that evolve with size. The design of atomically-defined samples with tunable size and shape is crucial in determining structure/properties correlations which eventually lead to optimized performance, as seen in many example discussed in this review. In addition to offer a superb control on the morphology of nanocrystalline materials, colloidal chemistry often permits the synthesis of materials unaccessible in their bulk form. Colloidal NCs can indeed be trapped in metastable crystalline phases thanks to the size-dependence of phase transformations (thermodynamics) and, above all, to the rather unique possibility of tuning the chemical potential of the reaction mixture by simply changing reactant concentration, temperature, precursors, surfactants (kinetics). As one example, the metastable $\mathrm{TiO}_{2}$ brookite phase was stabilized over a wide dimensional range (30$200 \mathrm{~nm}$ ) contrary to thermodynamic predictions (figure 1G) [9]. In another example, $\mathrm{V}_{2} \mathrm{O}_{3}$ in its metastable bixbyte phase was observed for the first time by exploiting the slow kinetics of transformation in the colloidal reaction mixture [136]. As another example already cited above, Bau et al. were able to synthesize Ni-Fe-O NCs in a wide compositional range in the metastable rock salt phase [119]. The access to phases which are not available in bulk could also open new opportunities in catalysis for water splitting. 


\subsection{Colloidal nanocrystals for water splitting: only model systems or technologically relevant systems?}

This review certainly evidences the role of colloidal NCs as excellent model systems. One question arises about whether colloidal NCs could become technologically relevant systems and which type of water-splitting reactor is better suited for such possibility. The implementation of colloidal NCs into various optoelectronic and electrochemical devices has been increasing over the last years. NC-based solar cells, flexible electronic circuits and, more recently, electrochromic windows and batteries have benefited from the NC solution processability and from the readily tunability of size, shape, crystalline phase and stoichiometries [4,21,137-139]. Devices for water electrolysis, based on electrochemical, photoelectrochemical and photocatalytic approaches, are emerging technologies [140]. Different reactor types have been considered, specifically particle suspension (baggie-type) reactors and panel arrays [2,31,141,142]. Single bed (type 1) and dual bed (type 2) baggie reactors have been proposed. In the former, each particle drives both OER and HER. The production of both $\mathrm{H}_{2}$ and $\mathrm{O}_{2}$ in the same vessel might constitute an explosive hazard, thus separation is needed. In the latter, one baggie performs OER and one baggie performs HER. A redox shuttle is required and its choice is crucial to reduce losses in the system. Panel arrays, fixed or tracking concentrator, are constituted by photoanode and photocathode thin films. Photovoltaic electrolysis is an alternative to such integrated photoelectrochemical devices [140,143]. The advantages of one versus the other have been discussed elsewhere [140,144,145]. Colloidal NCs can be assembled in electrodes/photoelectrodes or utilized in solution. A schematic representation of implementation of NCs in different reactor types is shown in figure 10. Progress still need to be made for an actual practical device based on colloidal NCs. For baggie-type reactors, the state-of-the art for the HNCs driving HER is quite advanced and record quantum yield of hydrogen generation up to $100 \%$ have been reached $[69,79]$. Yet, their activity under solar irradiation remains to be tested and their stability to be improved. As discussed in chapter 3, the slow transfer and subsequent reactions of the photoexcited holes and the ensuing high charge recombination rates often limit the efficiency of the photocatalysts. The use of hole scavengers with proper redox potential has been reported to improve the stability [67]. Also high $\mathrm{pH}$ has been reported to improve the stability of $\mathrm{CdSe} / \mathrm{Ni} \mathrm{NRs}$ system because of the redox couple ${ }^{-} \mathrm{OH} / \cdot \mathrm{OH}$ operating as a shuttle between the surface of the NCs and the ethanol scavenger in highly alkaline conditions [68,69].

No example relative to hybrid NCs for OER has been reported yet. Studies on redox shuttle are also far from being exhausted. As for thin film photoelectrodes assembled from NCs, new device architectures must be sought after to permit to fully benefit from the size-tunable properties of colloidal semiconductor NCs. At the same time, new metal oxide light absorbers need to be discovered, as discussed in the previous section. The easy processability of colloidal NCs should facilitate their integration in electrolyzers, once long term stability under operational conditions is demonstrated. 

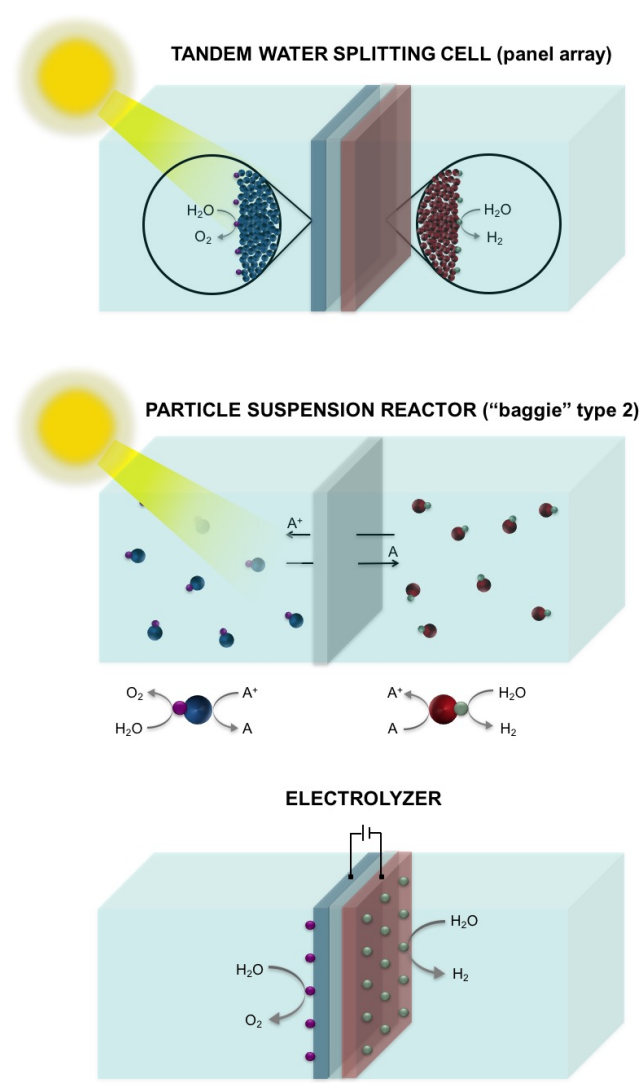

Figure 10. Schematic representation of colloidal NCs implemented in different reactor types for water splitting.

\section{Conclusions}

Herein, we have reviewed the utilization of colloidal NCs as light absorbers and electrocatalysts for water splitting. We have shown how the tunability of these materials is ideal to investigate the complex phenomena behind storing energy in chemical bonds and to optimize performance through structural and compositional modification. Nonetheless, the full potential of colloidal chemistry to advance studies of artificial photosynthesis is far from being fully explored. In this review, the emerging challenge of electrochemical $\mathrm{CO}_{2}$ reduction has not been discussed. Yet, there are already many examples in the literature which illustrate how colloidal chemistry can aid to reveal design guidelines for producing new catalysts with improved activity and selectivity $[20,146,147]$. The superb control offered by colloidal chemistry is rapidly expanding towards NC-based multifunctional hybrid materials, where colloidal NCs are interfaced with chemically diverse building blocks, such as polymers or glass matrixes $[139,148]$. These hybrids are expected to satisfy the demand for complexity of energy devices which is hard to meet by single component materials, thus they might open opportunities to uncover novel phenomena in artificial photosynthesis. As the interest increases and more research groups dedicate their effort to this field, basic research will be key to discover new materials and to propose new device architectures. At the same time, more applied studies will aid to demonstrate the effective utility of colloidal NCs in technologicallyrelevant photoelectrochemical and electrochemical reactors. 


\section{Acknowledgements}

We thank our home institution (EPFL), the Swiss Science National Foundation (AP Energy Grant, project number PYAPP2_166897/1) and the European Union (FP7 ERC Starting Grant "HY-CAT" - just awarded). A.L acknowledges the H2020-Marie Curie Individual Fellowship with grant agreement number 701745 for financial support.

[1] Fujishima A and Honda K 1972 Photolysis-decomposition of water at the surface of an irradiated semiconductor Nature 238 37-8

[2] Pinaud B A, Benck J D, Seitz L C, Forman A J, Chen Z, Deutsch T G, James B D, Baum K N, Baum G N, Ardo S, Wang H, Miller E and Jaramillo T F 2013 Technical and economic feasibility of centralized facilities for solar hydrogen production via photocatalysis and photoelectrochemistry Energy Environ. Sci. 6 1983

[3] Hu S, Xiang C, Haussener S, Berger A D and Lewis N S 2013 An analysis of the optimal band gaps of light absorbers in integrated tandem photoelectrochemical water-splitting systems Energy Environ. Sci. 6 2984-93

[4] Kovalenko M V, Manna L, Cabot A, Hens Z, Talapin D V, Kagan C R, Klimov V I, Rogach A L, Reiss P and Milliron D J 2015 Prospects of nanoscience with nanocrystals ACS Nano 9 1012-57

[5] Yin Y and Alivisatos A P 2005 Colloidal nanocrystal synthesis and the organicinorganic interface. Nature 437 664-70

[6] Jun Y, Choi J and Cheon J 2006 Shape Control of Semiconductor and Metal Oxide Nanocrystals through Nonhydrolytic Colloidal Routes Angew. Chemie Int. Ed. 45 3414-39

[7] Lee S-M, Cho S-N and Cheon J 2003 Anisotropic Shape Control of Colloidal Inorganic Nanocrystals Adv. Mater. 15 441-4

[8] Jun Y, Lee J-H, Choi J and Cheon J 2005 Symmetry-Controlled Colloidal Nanocrystals: Nonhydrolytic Chemical Synthesis and Shape Determining Parameters J. Phys. Chem. B 109 14795-806

[9] Buonsanti R, Grillo V, Carlino E, Giannini C, Kipp T, Cingolani R and Cozzoli P D 2008 Nonhydrolytic Synthesis of High-Quality Anisotropically Shaped Brookite $\mathrm{TiO}_{2}$ Nanocrystals J. Am. Chem. Soc. 130 11223-33

[10] Hyeon T 2003 Chemical synthesis of magnetic nanoparticles Chem. Commun. 927-34

[11] de Mello Donegá C, Liljeroth P and Vanmaekelbergh D 2005 Physicochemical Evaluation of the Hot-Injection Method, a Synthesis Route for Monodisperse Nanocrystals Small 1 1152-62

[12] Kwon S G, Piao Y, Park J, Angappane S, Jo Y, Hwang N-M, Park J-G and Hyeon T 2007 Kinetics of Monodisperse Iron Oxide Nanocrystal Formation by "HeatingUp" Process J. Am. Chem. Soc. 129 12571-84

[13] Kobayashi M, Tomita K, Petrykin V, Yoshimura M and Kakihana M 2008 Direct synthesis of brookite-type titanium oxide by hydrothermal method using watersoluble titanium complexes J. Mater. Sci. 43 2158-62

[14] Conca E, Aresti M, Saba M, Casula M F, Quochi F, Mula G, Loche D, Kim M R, Manna L, Corrias A, Mura A and Bongiovanni G 2014 Charge separation in Ptdecorated CdSe@CdS octapod nanocrystals Nanoscale 6 2238-43 
[15] Buonsanti R, Grillo V, Carlino E, Giannini C, Gozzo F, Garcia-Hernandez M, Garcia M A, Cingolani R and Cozzoli P D 2010 Architectural Control of SeededGrown Magnetic- Semicondutor Iron Oxide- TiO2 Nanorod Heterostructures: The Role of Seeds in Topology Selection J. Am. Chem. Soc. 132 2437-64

[16] Loiudice A, Cooper J K, Hess L H, Mattox T M, Sharp I D and Buonsanti R 2015 Assembly and Photocarrier Dynamics of Heterostructured Nanocomposite Photoanodes from Multicomponent Colloidal Nanocrystals Nano Lett. 15 7347-54

[17] Hodges J M, Morse J R, Williams M E and Schaak R E 2015 Microscopic Investigation of Chemoselectivity in $\mathrm{Ag}-\mathrm{Pt}-\mathrm{Fe}_{3} \mathrm{O}_{4}$ Heterotrimer Formation: Mechanistic Insights and Implications for Controlling High-Order Hybrid Nanoparticle Morphology J. Am. Chem. Soc. 137 15493-500

[18] Vinokurov K, Bekenstein Y, Gutkin V, Popov I, Millo O and Banin U 2014 Rhodium growth on $\mathrm{Cu} 2 \mathrm{~S}$ nanocrystals yielding hybrid nanoscale inorganic cages and their synergistic properties CrystEngComm 16 9506-12

[19] Zhang J, Ji M, Liu J and Xu M 2016 Metal/Semiconductor Hybrid Nanocrystals and Synergistic Photocatalysis Applications Advanced Catalytic Materials Photocatalysis and Other Current Trends ed L E Norena and J-A Wang (InTech)

[20] Loiudice A, Lobaccaro P, Kamali E A, Thao T, Huang B H, Ager J W and Buonsanti R 2016 Tailoring Copper Nanocrystals towards $\mathrm{C}_{2}$ Products in Electrochemical $\mathrm{CO}_{2}$ Reduction Angew. Chemie Int. Ed. 55 5789-92

[21] Shavel A, Ibáñez M, Luo Z, De Roo J, Carrete A, Dimitrievska M, Genç A, Meyns M, Pérez-Rodríguez A, Kovalenko M V., Arbiol J and Cabot A 2016 Scalable Heating-Up Synthesis of Monodisperse $\mathrm{Cu}_{2} \mathrm{ZnSnS}_{4}$ Nanocrystals Chem. Mater. 28 720-6

[22] Nedelcu G, Protesescu L, Yakunin S, Bodnarchuk M I, Grotevent M J and Kovalenko M V. 2015 Fast Anion-Exchange in Highly Luminescent Nanocrystals of Cesium Lead Halide Perovskites $\left(\mathrm{CsPbX}_{3}, \mathrm{X}=\mathrm{Cl}\right.$, Br, I) Nano Lett. 15 563540

[23] Yarema O, Yarema M, Lin W M M and Wood V $2016 \mathrm{Cu}-\mathrm{In}-\mathrm{Te}$ and Ag-In-Te colloidal nanocrystals with tunable composition and size Chem. Commun. $\mathbf{5 2}$ 10878-81

[24] Buonsanti R, Llordes A, Aloni S, Helms B A and Milliron D J 2011 Tunable Infrared Absorption and Visible Transparency of Colloidal Aluminum-Doped Zinc Oxide Nanocrystals Nano Lett. 11 4706-10

[25] Ye X, Chen J, Engel M, Millan J A, Li W, Qi L, Xing G, Collins J E, Kagan C R, Li J, Glotzer S C and Murray C B 2013 Competition of shape and interaction patchiness for self-assembling nanoplates Nat Chem 5 466-73

[26] Yamada Y, Tsung C-K, Huang W, Huo Z, Habas S E, Soejima T, Aliaga C E, Somorjai G A and Yang P 2011 Nanocrystal bilayer for tandem catalysis Nat. Chem. 3 372-6

[27] Cargnello M, Doan-Nguyen V V T, Gordon T R, Diaz R E, Stach E A, Gorte R J, Fornasiero P and Murray C B 2013 Control of Metal Nanocrystal Size Reveals Metal-Support Interface Role for Ceria Catalysts Science (80-. ). 341

[28] Chen G, Zhao Y, Fu G, Duchesne P N, Gu L, Zheng Y, Weng X, Chen M, Zhang $\mathrm{P}$ and Pao C-W 2014 Interfacial effects in iron-nickel hydroxide-platinum nanoparticles enhance catalytic oxidation Science (80-. ). 344 495-9 
[29] Niu Z, Becknell N, Yu Y, Kim D, Chen C, Kornienko N, Somorjai G A and Yang P 2016 Anisotropic phase segregation and migration of Pt in nanocrystals en route to nanoframe catalysts Nat. Mater. 1 1-21

[30] Huang X, Zhao Z, Cao L, Chen Y, Zhu E, Lin Z, Li M, Yan A, Zettl A and Wang Y M 2015 High-performance transition metal-doped Pt3Ni octahedra for oxygen reduction reaction Science (80-. ). 348 1230-4

[31] Fabian D M, Hu S, Singh N, Houle F A, Hisatomi T, Domen K, Osterloh F E and Ardo S 2015 Particle suspension reactors and materials for solar-driven water splitting Energy Environ. Sci. 8 2825-50

[32] Compton O C, Mullet C H, Shirley Chiang A and Frank E. Osterloh 2008 A Building Block Approach to Photochemical Water-Splitting Catalysts Based on Layered Niobate Nanosheets

[33] Wang J, Zhao J and Osterloh F E 2015 Photochemical charge transfer observed in nanoscale hydrogen evolving photocatalysts using surface photovoltage spectroscopy Energy Environ. Sci. 8 2970-6

[34] Wang Q, Hisatomi T, Jia Q, Tokudome H, Zhong M, Wang C, Pan Z, Takata T, Nakabayashi M, Shibata N, Li Y, Sharp I D, Kudo A, Yamada T and Domen K 2016 Scalable water splitting on particulate photocatalyst sheets with a solar-tohydrogen energy conversion efficiency exceeding 1\% Nat Mater 15 611-5

[35] Ham Y, Minegishi T, Hisatomi T and Domen K $2016 \mathrm{~A} \mathrm{SrTiO}_{3}$ photoanode prepared by the particle transfer method for oxygen evolution from water with high quantum efficiencies Chem. Commun. 52 5011-4

[36] Cozzoli P D, Pellegrino T and Manna L 2006 Synthesis, properties and perspectives of hybrid nanocrystal structures Chem. Soc. Rev. 35 1195-208

[37] Buonsanti R and Milliron D J 2013 Chemistry of doped colloidal nanocrystals Chem. Mater. 25 1305-17

[38] Manna L and Kudera S 2008 Mechanisms underlying the growth of inorganic nanoparticles in the liquid phase Advanced Wet-Chemical Synthetic Approaches to Inorganic Nanostructures (Kerala, India: Transworld Research Network) pp 1-64

[39] Mokari T, Sztrum C G, Salant A, Rabani E and Banin U 2005 Formation of asymmetric one-sided metal-tipped semiconductor nanocrystal dots and rods Nat. Mater. 4 855-63

[40] Buonsanti R, Grillo V, Carlino E, Giannini C, Curri M L, Innocenti C, Sangregorio C, Achterhold K, Parak F G, Agostiano A and Cozzoli P D 2006 Seeded growth of asymmetric binary nanocrystals made of a semiconductor $\mathrm{TiO} 2$ rodlike section and a magnetic $\gamma$-Fe2O3 spherical domain J. Am. Chem. Soc. 128 16953-70

[41] Costi R, Saunders A E and Banin U 2010 Colloidal Hybrid Nanostructures: A New Type of Functional Materials Angew. Chemie Int. Ed. 49 4878-97

[42] Wu K and Lian T 2016 Quantum confined colloidal nanorod heterostructures for solar-to-fuel conversion Chem. Soc. Rev. 45 3781-810

[43] Kwon S G, Krylova G, Phillips P J, Klie R F, Chattopadhyay S, Shibata T, Bunel E E, Liu Y, Prakapenka V B, Lee B and Shevchenko E V 2015 Heterogeneous nucleation and shape transformation of multicomponent metallic nanostructures Nat Mater 14 215-23

[44] Frank F C and Van der Merwe J H 1948 One-dimensional dislocations. 1. Static 
theory Proc. R. Soc. London. Ser. A Math. Phys. Sci. 198205

[45] Volmer M and Weber A 1926 Keimbildung in übersättigten Gebilden Z. phys. Chem 119 277-301

[46] Stranski I N and Krastanow L 1937 Zur Theorie der orientierten Ausscheidung von Ionenkristallen aufeinander Monatshefte für Chemie 71 351-64

[47] Jin M, Zhang H, Wang J, Zhong X, Lu N, Li Z, Xie Z, Kim M J and Xia Y 2012 Copper Can Still Be Epitaxially Deposited on Palladium Nanocrystals To Generate Core-Shell Nanocubes Despite Their Large Lattice Mismatch ACS Nano 6 256673

[48] Han Z, Qiu F, Eisenberg R, Holland P L and Krauss T D 2012 Robust Photogeneration of $\mathrm{H} 2$ in Water Using Semiconductor Nanocrystals and a Nickel Catalyst Science (80-. ). 338 1321-4

[49] Brown K A, Wilker M B, Boehm M, Dukovic G and King P W 2012 Characterization of Photochemical Processes for $\mathrm{H}_{2}$ Production by CdS Nanorod[FeFe] Hydrogenase Complexes J. Am. Chem. Soc. 134 5627-36

[50] Brown K A, Dayal S, Ai X, Rumbles G and King P W 2010 Controlled Assembly of Hydrogenase-CdTe Nanocrystal Hybrids for Solar Hydrogen Production J. Am. Chem. Soc. 132 9672-80

[51] Patra B K, Khilari S, Pradhan D and Pradhan N 2016 Hybrid Dot-Disk Au-CuInS ${ }_{2}$ Nanostructures as Active Photocathode for Efficient Evolution of Hydrogen from Water Chem. Mater. 28 4358-66

[52] Korala L, Wang Z, Liu Y, Maldonado S and Brock S L 2013 Uniform Thin Films of CdSe and CdSe(ZnS) Core(Shell) Quantum Dots by Sol-Gel Assembly: Enabling Photoelectrochemical Characterization and Electronic Applications ACS Nano 7 1215-23

[53] Ben-Shahar Y and Banin U 2016 Hybrid Semiconductor-Metal Nanorods as Photocatalysts Top. Curr. Chem. 37454

[54] Wilker M B, Schnitzenbaumer K J and Dukovic G 2012 Recent Progress in Photocatalysis Mediated by Colloidal II-VI Nanocrystals Isr. J. Chem. 52 1002-15

[55] Wu K and Lian T 2016 Quantum confined colloidal nanorod heterostructures for solar-to-fuel conversion Chem. Soc. Rev. 45 3781-810

[56] Chen X, Shen S, Guo L and Mao S S 2010 Semiconductor-based Photocatalytic Hydrogen Generation Chem. Rev. 110 6503-70

[57] Zhao W, Huang Y, Liu Y, Cao L, Zhang F, Guo Y and Zhang B 2016 A Heterogeneous Photocatalytic Hydrogen Evolution Dyad: [(tpy Chem. - A Eur. J.

[58] Berr M, Vaneski A, Susha A S, Rodríguez-Fernández J, Döblinger M, Jäckel F, Rogach A L and Feldmann J 2010 Colloidal CdS nanorods decorated with subnanometer sized Pt clusters for photocatalytic hydrogen generation Appl. Phys. Lett. 9793108

[59] Sung Y, Lim J, Koh J H, Hill L J, Min B K, Pyun J and Char K 2015 Uniform decoration of Pt nanoparticles on well-defined CdSe tetrapods and the effect of their Pt cluster size on photocatalytic H 2 generation CrystEngComm 17 8423-7

[60] Kalisman P, Houben L, Aronovitch E, Kauffmann Y, Bar-Sadan M and Amirav L 2015 The golden gate to photocatalytic hydrogen production J. Mater. Chem. A 3 19679-82

[61] Ben-Shahar Y, Scotognella F, Waiskopf N, Kriegel I, Dal Conte S, Cerullo G and 
Banin U 2015 Effect of Surface Coating on the Photocatalytic Function of Hybrid CdS-Au Nanorods Small 11 462-71

[62] Ben-Shahar Y, Scotognella F, Kriegel I, Moretti L, Cerullo G, Rabani E and Banin U 2016 Optimal metal domain size for photocatalysis with hybrid semiconductor-metal nanorods Nat. Commun. 710413

[63] Cao S, Chen Y, Hou C-C, Lv X-J and Fu W-F 2015 Cobalt phosphide as a highly active non-precious metal cocatalyst for photocatalytic hydrogen production under visible light irradiation J. Mater. Chem. A 3 6096-101

[64] Cao S, Chen Y, Wang C-J, He P and Fu W-F 2014 Highly efficient photocatalytic hydrogen evolution by nickel phosphide nanoparticles from aqueous solution Chem. Commun. 50 10427-9

[65] Zhukovskyi M, Tongying P, Yashan H, Wang Y and Kuno M 2015 Efficient Photocatalytic Hydrogen Generation from Ni Nanoparticle Decorated CdS Nanosheets ACS Catal. 5 6615-23

[66] Cheng H, Lv X-J, Cao S, Zhao Z-Y, Chen Y and Fu W-F 2016 Robustly photogenerating $\mathrm{H} 2$ in water using $\mathrm{FeP} / \mathrm{CdS}$ catalyst under solar irradiation. Sci. Rep. 619846

[67] Berr M J, Wagner P, Fischbach S, Vaneski A, Schneider J, Susha A S, Rogach A L, Jäckel F and Feldmann J 2012 Hole scavenger redox potentials determine quantum efficiency and stability of Pt-decorated $\mathrm{CdS}$ nanorods for photocatalytic hydrogen generation Appl. Phys. Lett. 100223903

[68] Schneider J, Vaneski A, Pesch G R, Susha A S, Yang Teoh W and Rogach A L 2014 Enhanced hydrogen evolution rates at high $\mathrm{pH}$ with a colloidal cadmium sulphide-platinum hybrid system APL Mater. 2126102

[69] Simon T, Bouchonville N, Berr M J, Vaneski A, Adrović A, Volbers D, Wyrwich R, Döblinger M, Susha A S, Rogach A L, Jäckel F, Stolarczyk J K and Feldmann J 2014 Redox shuttle mechanism enhances photocatalytic H2 generation on Nidecorated CdS nanorods. Nat. Mater. 13 1013-8

[70] Amirav L and Alivisatos A P 2010 Photocatalytic Hydrogen Production with Tunable Nanorod Heterostructures J. Phys. Chem. Lett. 1 1051-4

[71] Wu K, Chen Z, Lv H, Zhu H, Hill C L and Lian T 2014 Hole Removal Rate Limits Photodriven $\mathrm{H}_{2}$ Generation Efficiency in CdS-Pt and CdSe/CdS-Pt Semiconductor Nanorod-Metal Tip Heterostructures J. Am. Chem. Soc. $1367708-$ 16

[72] Olshansky J H, Ding T X, Lee Y V., Leone S R and Alivisatos A P 2015 Hole Transfer from Photoexcited Quantum Dots: The Relationship between Driving Force and Rate J. Am. Chem. Soc. 137 15567-75

[73] Tarafder K, Surendranath Y, Olshansky J H, Alivisatos A P and Wang L-W 2014 Hole Transfer Dynamics from a CdSe/CdS Quantum Rod to a Tethered Ferrocene Derivative J. Am. Chem. Soc. 136 5121-31

[74] Nakibli Y and Amirav L 2016 Selective Growth of Ni Tips on Nanorod Photocatalysts Chem. Mater. 28 4524-7

[75] Aronovitch E, Kalisman P, Mangel S, Houben L, Amirav L and Bar-Sadan M 2015 Designing Bimetallic Co-Catalysts: A Party of Two J. Phys. Chem. Lett. 6 3760-4

[76] Yu P, Wen X, Lee Y-C, Lee W-C, Kang C-C and Tang J 2013 Photoinduced 
Ultrafast Charge Separation in Plexcitonic CdSe/Au and CdSe/Pt Nanorods $J$. Phys. Chem. Lett. 4 3596-601

[77] Aronovitch E, Kalisman P, Houben L, Amirav L and Bar-Sadan M 2016 Stability of Seeded Rod Photocatalysts: Atomic Scale View Chem. Mater. 28 1546-52

[78] Ehrat F, Simon T, Stolarczyk J K and Feldmann J 2015 Size Effects on Photocatalytic H2 Generation with CdSe/CdS Core-Shell Nanocrystals Zeitschrift für Phys. Chemie 229 205-19

[79] Kalisman P, Nakibli Y and Amirav L 2016 Perfect Photon-to-Hydrogen Conversion Efficiency Nano Lett. 16 1776-81

[80] Acharya K P, Khnayzer R S, O'Connor T, Diederich G, Kirsanova M, Klinkova A, Roth D, Kinder E, Imboden M and Zamkov M 2011 The Role of Hole Localization in Sacrificial Hydrogen Production by Semiconductor-Metal Heterostructured Nanocrystals Nano Lett. 11 2919-26

[81] Berr M J 2012 Hole scavenger redox potentials determine quantum efficiency and stability of Pt-decorated CdS nanorods for photocatalytic hydrogen generation Appl. Phys. Lett. 100

[82] Chen Y, Zhao S, Wang X, Peng Q, Lin R, Wang Y, Shen R, Cao X, Zhang L, Zhou G, Li J, Xia A and Li Y 2016 Synergetic Integration of $\mathrm{Cu}_{1.94} \mathrm{~S}-\mathrm{Zn}_{x} \mathrm{Cd}_{1-}$ ${ }_{x}$ S Heteronanorods for Enhanced Visible-Light-Driven Photocatalytic Hydrogen Production J. Am. Chem. Soc. 138 4286-9

[83] Yu X, Shavel A, An X, Luo Z, Ibáñez M and Cabot A $2014 \mathrm{Cu}_{2} \mathrm{ZnSnS}_{4}$-Pt and $\mathrm{Cu}_{2} \mathrm{ZnSnS}_{4}-\mathrm{Au}$ Heterostructured Nanoparticles for Photocatalytic Water Splitting and Pollutant Degradation J. Am. Chem. Soc. 136 9236-9

[84] Yu X, An X, Shavel A, Ibáñez M and Cabot A 2014 The effect of the Ga content on the photocatalytic hydrogen evolution of CuIn 1-x Ga x S 2 nanocrystals $J$. Mater. Chem. A 2 12317-22

[85] Regulacio M D and Han M-Y 2016 Multinary I-III-VI 2 and I 2 -II-IV-VI 4 Semiconductor Nanostructures for Photocatalytic Applications Acc. Chem. Res. 49 $511-9$

[86] Ye C, Regulacio M D, Lim S H, Li S, Xu Q-H and Han M-Y 2015 Alloyed ZnS$\mathrm{CuInS}{ }_{2}$ Semiconductor Nanorods and Their Nanoscale Heterostructures for Visible-Light-Driven Photocatalytic Hydrogen Generation Chem. - A Eur. J. 21 9514-9

[87] Kalisman P, Kauffmann Y and Amirav L 2015 Photochemical oxidation on nanorod photocatalysts J. Mater. Chem. A 3 3261-5

[88] Amirav L, Oba F, Aloni S and Alivisatos A P 2015 Modular Synthesis of a Dual Metal-Dual Semiconductor Nano-Heterostructure Angew. Chemie Int. Ed. 54 7007-11

[89] Mainz R, Singh A, Levcenko S, Klaus M, Genzel C, Ryan K M and Unold T 2014 Phase-transition-driven growth of compound semiconductor crystals from ordered metastable nanorods Nat Commun $\mathbf{5}$

[90] Lynch J, Giannini C, Cooper J K, Loiudice A, Sharp I D and Buonsanti R 2015 Substitutional or Interstitial Site-Selective Nitrogen Doping in $\mathrm{TiO}_{2}$ Nanostructures J. Phys. Chem. C 119 7443-52

[91] Gonçalves R H, Leite L D T and Leite E R 2012 Colloidal WO 3 Nanowires as a Versatile Route to Prepare a Photoanode for Solar Water Splitting ChemSusChem 


\section{$52341-7$}

[92] Hilaire S, Süess M J, Kränzlin N, Bieńkowski K, Solarska R, Augustyński J and Niederberger M 2014 Microwave-assisted nonaqueous synthesis of WO 3 nanoparticles for crystallographically oriented photoanodes for water splitting $J$. Mater. Chem. A 2 20530-7

[93] Gonçalves R H, Lima B H R and Leite E R 2011 Magnetite Colloidal Nanocrystals: A Facile Pathway To Prepare Mesoporous Hematite Thin Films for Photoelectrochemical Water Splitting J. Am. Chem. Soc. $1336012-9$

[94] Gonçalves R H and Leite E R 2014 The colloidal nanocrystal deposition process: an advanced method to prepare high performance hematite photoanodes for water splitting Energy Environ. Sci. 7 2250-4

[95] Wang J-J, Hu Y, Toth R, Fortunato G and Braun A 2016 A facile nonpolar organic solution process of a nanostructured hematite photoanode with high efficiency and stability for water splitting J. Mater. Chem. A 4 2821-5

[96] Wang J, Zhang N, Su J and Guo L $2016 \alpha$-Fe 2 O 3 quantum dots: low-cost synthesis and photocatalytic oxygen evolution capabilities $R S C A d v$. 6 41060-6

[97] Abdi F F, Han L, Smets A H M, Zeman M, Dam B and van de Krol R 2013 Efficient solar water splitting by enhanced charge separation in a bismuth vanadate-silicon tandem photoelectrode Nat. Commun. 4

[98] Kim T W and Choi K-S 2014 Nanoporous BiVO4 Photoanodes with Dual-Layer Oxygen Evolution Catalysts for Solar Water Splitting Science (80-. ). 343 990-4

[99] Shi X, Choi I Y, Zhang K, Kwon J, Kim D Y, Lee J K, Oh S H, Kim J K and Park J H 2014 Efficient photoelectrochemical hydrogen production from bismuth vanadate-decorated tungsten trioxide helix nanostructures Nat. Commun. 54775

[100] Bretos I, Jiménez R, Wu A, Kingon A I, Vilarinho P M and Calzada M L 2014 Activated Solutions Enabling Low-Temperature Processing of Functional Ferroelectric Oxides for Flexible Electronics Adv. Mater. 26 1405-9

[101] Bretos I, Jiménez R, Pérez-Mezcua D, Salazar N, Ricote J and Calzada M L 2015 Low-Temperature Liquid Precursors of Crystalline Metal Oxides Assisted by Heterogeneous Photocatalysis Adv. Mater. 27 2608-13

[102] Loiudice A, Ma J, Drisdell W S, Mattox T M, Cooper J K, Thao T, Giannini C, Yano J, Wang L-W, Sharp I D and Buonsanti R 2015 Bandgap Tunability in SbAlloyed BiVO ${ }_{4}$ Quaternary Oxides as Visible Light Absorbers for Solar Fuel Applications $A d v$. Mater. 27 6733-40

[103] McCrory C C L, Jung S, Ferrer I M, Chatman S M, Peters J C and Jaramillo T F 2015 Benchmarking Hydrogen Evolving Reaction and Oxygen Evolving Reaction Electrocatalysts for Solar Water Splitting Devices J. Am. Chem. Soc. 137 4347-57

[104] McCrory C C L, Jung S, Peters J C and Jaramillo T F 2013 Benchmarking Heterogeneous Electrocatalysts for the Oxygen Evolution Reaction J. Am. Chem. Soc. 135 16977-87

[105] Callejas J F, Read C G, Roske C W, Lewis N S and Schaak R E 2016 Synthesis, Characterization, and Properties of Metal Phosphide Catalysts for the HydrogenEvolution Reaction Chem. Mater. 28 6017-44

[106] Popczun E J, McKone J R, Read C G, Biacchi A J, Wiltrout A M, Lewis N S and Schaak R E 2013 Nanostructured Nickel Phosphide as an Electrocatalyst for the Hydrogen Evolution Reaction J. Am. Chem. Soc. $1359267-70$ 
[107] Seo B, Baek D S, Sa Y J and Joo S H 2016 Shape effects of nickel phosphide nanocrystals on hydrogen evolution reaction CrystEngComm 18 6083-9

[108] Zhou M, Kang Y, Huang K, Shi Z, Xie R and Yang W 2016 Ultra-small nickel phosphide nanoparticles as a high-performance electrocatalyst for the hydrogen evolution reaction $R S C A d v .6$ 74895-902

[109] Popczun E J, Read C G, Roske C W, Lewis N S and Schaak R E 2014 Highly Active Electrocatalysis of the Hydrogen Evolution Reaction by Cobalt Phosphide Nanoparticles Angew. Chemie 126 5531-4

[110] Callejas J F, Read C G, Popczun E J, McEnaney J M and Schaak R E 2015 Nanostructured $\mathrm{Co}_{2} \mathrm{P}$ Electrocatalyst for the Hydrogen Evolution Reaction and Direct Comparison with Morphologically Equivalent CoP Chem. Mater. 27 376974

[111] Popczun E J, Roske C W, Read C G, Crompton J C, McEnaney J M, Callejas J F, Lewis N S and Schaak R E 2015 Highly branched cobalt phosphide nanostructures for hydrogen-evolution electrocatalysis J. Mater. Chem. A 3 5420-5

[112] Ha D-H, Han B, Risch M, Giordano L, Yao K P C, Karayaylali P and Shao-Horn Y 2016 Activity and stability of cobalt phosphides for hydrogen evolution upon water splitting Nano Energy

[113] Callejas J F, McEnaney J M, Read C G, Crompton J C, Biacchi A J, Popczun E J, Gordon T R, Lewis N S and Schaak R E 2014 Electrocatalytic and Photocatalytic Hydrogen Production from Acidic and Neutral-pH Aqueous Solutions Using Iron Phosphide Nanoparticles ACS Nano 8 11101-7

[114] McEnaney J M, Chance Crompton J, Callejas J F, Popczun E J, Read C G, Lewis N S and Schaak R E 2014 Electrocatalytic hydrogen evolution using amorphous tungsten phosphide nanoparticles Chem. Commun. 50 11026-8

[115] McEnaney J M, Crompton J C, Callejas J F, Popczun E J, Biacchi A J, Lewis N S and Schaak R E 2014 Amorphous Molybdenum Phosphide Nanoparticles for Electrocatalytic Hydrogen Evolution Chem. Mater. 26 4826-31

[116] McEnaney J M, Soucy T L, Hodges J M, Callejas J F, Mondschein J S and Schaak R E 2016 Colloidally-synthesized cobalt molybdenum nanoparticles as active and stable electrocatalysts for the hydrogen evolution reaction under alkaline conditions J. Mater. Chem. A 4 3077-81

[117] Fominykh K, Chernev P, Zaharieva I, Sicklinger J, Stefanic G, Döblinger M, Müller A, Pokharel A, Böcklein S, Scheu C, Bein T and Fattakhova-Rohlfing D 2015 Iron-Doped Nickel Oxide Nanocrystals as Highly Efficient Electrocatalysts for Alkaline Water Splitting ACS Nano 9 5180-8

[118] Bau J A, Li P, Marenco A J, Trudel S, Olsen B C, Luber E J and Buriak J M 2014 Nickel/Iron Oxide Nanocrystals with a Nonequilibrium Phase: Controlling Size, Shape, and Composition Chem. Mater. 26 4796-804

[119] Bau J A, Luber E J and Buriak J M 2015 Oxygen Evolution Catalyzed by NickelIron Oxide Nanocrystals with a Nonequilibrium Phase ACS Appl. Mater. Interfaces 7 19755-63

[120] Masud J, Umapathi S, Ashokaan N and Nath M 2016 Iron phosphide nanoparticles as an efficient electrocatalyst for the OER in alkaline solution $J$. Mater. Chem. A 4 9750-4

[121] Dutta A, Samantara A K, Dutta S K, Jena B K and Pradhan N 2016 Surface- 
Oxidized Dicobalt Phosphide Nanoneedles as a Nonprecious, Durable, and Efficient OER Catalyst ACS Energy Lett. 1 169-74

[122] Stern L-A, Feng L, Song F and Hu X 2015 Ni 2 P as a Janus catalyst for water splitting: the oxygen evolution activity of $\mathrm{Ni} 2 \mathrm{P}$ nanoparticles Energy Environ. Sci. 8 2347-51

[123] Li D, Baydoun H, Verani C N and Brock S L 2016 Efficient Water Oxidation Using CoMnP Nanoparticles J. Am. Chem. Soc. 138 4006-9

[124] Boles M A, Ling D, Hyeon T and Talapin D V 2016 The surface science of nanocrystals Nat Mater 15 141-53

[125] De Roo J, Van Driessche I, Martins J C and Hens Z 2016 Colloidal metal oxide nanocrystal catalysis by sustained chemically driven ligand displacement $\mathrm{Nat}$ Mater 15 517-21

[126] Garcia G, Buonsanti R, Llordes A, Runnerstrom E L, Bergerud A and Milliron D J 2013 Near-Infrared Spectrally Selective Plasmonic Electrochromic Thin Films Adv. Opt. Mater. $1215-20$

[127] Rosen E L, Buonsanti R, Llordes A, Sawvel A M, Milliron D J and Helms B A 2012 Exceptionally Mild Reactive Stripping of Native Ligands from Nanocrystal Surfaces by Using Meerwein's Salt Angew. Chemie Int. Ed. 51 684-9

[128] Dong A, Ye X, Chen J, Kang Y, Gordon T, Kikkawa J M and Murray C B 2011 A generalized ligand-exchange strategy enabling sequential surface functionalization of colloidal nanocrystals. J. Am. Chem. Soc. 133 998-1006

[129] Kovalenko M V, Scheele M and Talapin D V 2009 Colloidal nanocrystals with molecular metal chalcogenide surface ligands. Science 324 1417-20

[130] Ning Z 2014 Air-stable n-type colloidal quantum dot solids Nat. Mater. 13

[131] Mistry H, Varela A S, Bonifacio C S, Zegkinoglou I, Sinev I, Choi Y-W, Kisslinger K, Stach E A, Yang J C, Strasser P and Cuenya B R 2016 Highly selective plasma-activated copper catalysts for carbon dioxide reduction to ethylene Nat Commun 7

[132] Wang P, Zhang J, He H, Xu X and Jin Y 2015 The important role of surface ligand on $\mathrm{CdSe} / \mathrm{CdS}$ core/shell nanocrystals in affecting the efficiency of $\mathrm{H}_{2}$ photogeneration from water Nanoscale 7 5767-75

[133] Ding T X, Olshansky J H, Leone S R and Alivisatos A P 2015 Efficiency of Hole Transfer from Photoexcited Quantum Dots to Covalently Linked Molecular Species J. Am. Chem. Soc. 137 2021-9

[134] Cao Z, Kim D, Hong D, Yu Y, Xu J, Lin S, Wen X, Nichols E M, Jeong K, Reimer J A, Yang P and Chang C J 2016 A Molecular Surface Functionalization Approach to Tuning Nanoparticle Electrocatalysts for Carbon Dioxide Reduction J. Am. Chem. Soc. 138 8120-5

[135] Chen T and Rodionov V O 2016 Controllable Catalysis with Nanoparticles: Bimetallic Alloy Systems and Surface Adsorbates ACS Catal. 6 4025-33

[136] Bergerud A, Buonsanti R, Jordan-Sweet J L and Milliron D J 2013 Synthesis and Phase Stability of Metastable Bixbyite $\mathrm{V}_{2} \mathrm{O}_{3}$ Colloidal Nanocrystals Chem. Mater. 25 3172-9

[137] Carey G H, Abdelhady A L, Ning Z, Thon S M, Bakr O M and Sargent E H 2015 Colloidal Quantum Dot Solar Cells Chem. Rev. 115 12732-63

[138] Kim D K, Lai Y, Diroll B T, Murray C B and Kagan C R 2012 Flexible and low- 
voltage integrated circuits constructed from high-performance nanocrystal transistors Nat Commun 31216

[139] Llordés A, Garcia G, Gazquez J and Milliron D J 2013 Tunable near-infrared and visible-light transmittance in nanocrystal-in-glass composites. Nature 500 323-6

[140] Lewis N S 2016 Research opportunities to advance solar energy utilization Science (80-.). 351

[141] Tachibana Y, Vayssieres L and Durrant J R 2012 Artificial photosynthesis for solar water-splitting Nat Phot. 6 511-8

[142] Abe R 2010 Recent progress on photocatalytic and photoelectrochemical water splitting under visible light irradiation J. Photochem. Photobiol. C Photochem. Rev. 11 179-209

[143] Xiang C, Papadantonakis K M and Lewis N S 2016 Principles and implementations of electrolysis systems for water splitting Mater. Horizons 3 16973

[144] Zhang K, Ma M, Li P, Wang D H and Park J H 2016 Water Splitting Progress in Tandem Devices: Moving Photolysis beyond Electrolysis Adv. Energy Mater. 6 1600602

[145] Jacobsson T J, Fjällström V, Edoff M and Edvinsson T 2014 Sustainable solar hydrogen production: from photoelectrochemical cells to PV-electrolyzers and back again Energy Environ. Sci. 7 2056-70

[146] Kim D, Resasco J, Yu Y, Asiri A M and Yang P 2014 Synergistic geometric and electronic effects for electrochemical reduction of carbon dioxide using goldcopper bimetallic nanoparticles Nat Commun 5

[147] Manthiram K, Beberwyck B J and Alivisatos A P 2014 Enhanced Electrochemical Methanation of Carbon Dioxide with a Dispersible Nanoscale Copper Catalyst $J$. Am. Chem. Soc. 136 13319-25

[148] Jeon K-J, Moon H R, Ruminski A M, Jiang B, Kisielowski C, Bardhan R and Urban J J 2011 Air-stable magnesium nanocomposites provide rapid and highcapacity hydrogen storage without using heavy-metal catalysts Nat Mater 10286 90 